\title{
Stellar hydrodynamics caught in the act: Asteroseismology with CoRoT and Kepler
}

\author{
Jørgen Christensen-Dalsgaard ${ }^{1}$ and Michael J. Thompson ${ }^{2,3}$ \\ ${ }^{1}$ Department of Physics and Astronomy, Aarhus University, 8000 Aarhus C, Denmark \\ email: jcd@phys.au.dk \\ ${ }^{2}$ School of Mathematics \& Statistics, University of Sheffield, Sheffield, S3 7RH, UK \\ ${ }^{3}$ High Altitude Observatory, National Center for Atmospheric Research, P.O. Box 3000, \\ Boulder, CO 80307-3000, USA \\ email: mjt@ucar.edu
}

\begin{abstract}
Asteroseismic investigations, particularly based on data on stellar oscillations from the CoRoT and Kepler space missions, are providing unique possibilities for investigating the properties of stellar interiors. This constitutes entirely new ways to study the effects of dynamic phenomena on stellar structure and evolution. Important examples are the extent of convection zones and the associated mixing and the direct and indirect effects of stellar rotation. In addition, the stellar oscillations themselves show very interesting dynamic behaviour. Here we discuss examples of the results obtained from such investigations, across the Hertzsprung-Russell diagram.
\end{abstract}

Keywords. hydrodynamics, asteroseismology, space vehicles, stars: interiors, stars: evolution, stars: oscillations, planetary systems

\section{Introduction}

Stellar interiors are obvious sites for interesting dynamical phenomena, with strong potential effects of convection and other forms of instabilities, rotation and its evolution, as well as magnetic fields, forming a fascinating playing field for theoretical investigations. However, 'classical' observations of stellar surfaces provide only limited observational tests of the resulting models. Observations of stellar oscillations, on the other hand, are sensitive to many aspects of the structure and dynamics of stellar interiors. In addition, the oscillations are themselves interesting dynamical phenomena. The diagnostic potential of oscillations has been evident for more than a decade in the solar case, where helioseismic investigations have yielded very detailed information about the structure and rotation of the solar interior (e.g., Gough \& Toomre 1991; Christensen-Dalsgaard 2002) and the detailed properties of the solar near-surface region through local helioseismology (for a review, see Gizon et al. 2010). For a comprehensive review of global helioand asteroseismology, see also Aerts et al. (2010).

The extension of such detailed investigations to other stars has been eagerly sought since the beginning of helioseismology, given the expected general presence of the oscillations in all cool stars. There is strong evidence that the solar modes are excited stochastically by the near-surface convection, and hence similar modes are expected in all stars with such vigorous convective motions (Christensen-Dalsgaard \& Frandsen 1983). However, the predicted amplitudes of a few parts per million in relative intensity or at most tens of centimeters per second in velocity have made their detection extremely challenging. Ground-based observations have had some success for a limited number of stars (for early examples, see Kjeldsen et al. 1995; Bouchy \& Carrier 2001, 2002; Frandsen et al. 
2002), but the required efforts in terms of manpower and valuable telescope time have been very considerable. However, in the last few years observations of stellar oscillations, and other types of variability, have made tremendous progress, largely as a result of the CoRoT and Kepler space missions, with significant contributions also from the Canadian MOST satellite (Walker et al. 2003; Matthews 2007). Here we provide a brief overview of some of the results from these missions which promise to revolutionize the study of stellar internal structure and dynamics.

\section{CoRoT and Kepler}

Both CoRoT and Kepler carry out photometric observations, with the dual purpose of detecting extra-solar planets (exoplanets) using the transit technique and making asteroseismic investigations. In fact, the observational requirements for these two types of investigation are very similar. A central goal of the exoplanet studies is to characterize the population of Earth-like planets in orbit around Sun-like stars. The corresponding transit corresponds to a reduction in stellar intensity by around $10^{-4}$. This level of sensitivity allows the detection of solar-like oscillations in sufficiently extended observations. Also, both types of investigations require very long and continuous observations. Finally, asteroseismology has the potential to characterize the properties of the central stars in planetary systems detected by the transit technique. This has proven to be very useful in a few cases for the Kepler mission and is central to the PLATO mission proposed to ESA.

The CoRoT satellite (Baglin et al. 2006, 2009; Michel et al. 2008a) was launched on 27 December 2006 into an orbit around the Earth. The satellite has an off-axis telescope with a diameter of $28 \mathrm{~cm}$, and a focal plane with 4 CCD detectors, two of which (defining the exoplanet field) are optimized for studying planet transits and the other two, with slightly defocussed images, optimized for asteroseismology (the asteroseismic field). The field of view of each CCD is $1.3^{\circ} \times 1.3^{\circ}$. Given the low-Earth orbit, great care was taken to minimize effects of scattered light. Further details on the design and operations of the mission were provided by Auvergne et al. (2009).

CoRoT's orbit allows extended observations, up to 5 months, in two regions with a diameter of around $20^{\circ}$, near the Galactic plane and the direction of the Galactic centre and anticentre, respectively; these are known as the 'CoRoT eyes'. The observed fields are selected within these regions, such that the asteroseismic detectors contain a suitable sample of relatively bright asteroseismic targets, up to a total of 10 targets, with an adequate density of stars in the corresponding exo field. It should be noted that while the observations in the latter are probably not sufficiently sensitive to study solar-like oscillations in main-sequence stars, they do provide very extensive data on other types of stellar variability, including solar-like oscillations in red giants (see Section 8). The photometric analysis is carried out on the satellite, with only the photometric signal being transmitted to the ground; a typical cadence for the astero field is $32 \mathrm{~s}$.

The mission suffered a loss of two of the four CCD detectors (one each in the exo and astero fields) on 8 March $2009 \dagger$ but is otherwise performing flawlessly. Early results on solar-like oscillations from CoRoT data were presented by Michel et al. (2008b). The mission has now been extended until March 2013.

The Kepler mission (Borucki et al. 2009; Koch et al. 2010) was launched on 7 March 2009 into an Earth-trailing heliocentric orbit, with a period of around 53 weeks. This provides a very stable environment for the observations, in terms of stray light and

$\dagger$ Coincidentally a day after the launch of the Kepler mission! 
other disturbances, although with the drawback of a gradually decreasing rate of data transmission with the increasing distance from Earth. Even so, it is hoped to keep the mission operating well beyond the current nominal lifetime of $3 \frac{1}{2}$ years. The Kepler photometer consists of a Schmidt telescope with a corrector diameter of $95 \mathrm{~cm}$ and a $16^{\circ}$ diameter field of view. The detector at the curved focal plane has 42 CCDs with a total of 95 megapixels. This provides a field of around 105 square degrees. The data are downlinked in the form of small images around each of the target stars, with the detailed photometry being carried out on the ground. This allows up to 170,000 targets to be observed at a 30-minute cadence, while up to 512 stars can be observed at a 1-minute cadence; the latter are the prime targets for asteroseismology, although for more slowly varying stars the long-cadence data are also extremely valuable. The spacecraft is rotated by $90^{\circ}$ four times per orbit to keep the solar arrays pointed towards the Sun. Thus the observations are naturally divided into quarters. New target lists are uploaded for each quarter, although targets can be observed for as little as one month each; typically, most targets are in fact observed for several quarters, in many cases throughout the mission. For further details on the spacecraft and the operations, see Koch et al. (2010).

A detector module, corresponding to two of the 42 CCD detectors, failed in January 2010. Otherwise, the mission has been operating successfully, reaching very close to the expected performance. Borucki et al. (2010) provided an early overview of Kepler results on exoplanets.

Kepler observes a fixed field in the region of the constellations of Cygnus and Lyra, centred $13.5^{\circ}$ above the Galactic plane and chosen to secure a sufficient number of targets of the right type while avoiding excessive problems with background confusion. A very detailed characterization of the field was carried out before launch, resulting in the Kepler Input Catalog (KIC; Brown, T. M., et al. 2011). To avoid problems with highly saturated trailing images, the field is located such that the brightest stars are placed in gaps between the CCDs. In addition, the CCDs are positioned such that a star is located at approximately the same point on a CCD following each quarterly rotation.

Kepler asteroseismology (e.g., Christensen-Dalsgaard et al. 2008) is carried out in the Kepler Asteroseismic Science Consortium (KASC), which at the time of writing has around 450 members. The members are organized into 13 working groups, generally dealing with different classes of pulsating stars. The KASC is responsible for proposing targets, and for the analysis and publication of the results. Data for the KASC are made available through the Kepler Asteroseismic Science Operations Centre (KASOC) in Aarhus, Denmark, which also organizes the sharing and discussion of manuscripts before publication. The structure of the Kepler Asteroseismic Investigation (KAI) was presented by Kjeldsen et al. (2010).

In the early phases of the KAI a survey was made of a very large number of stars, to characterize their oscillation properties and provide the basis for selecting targets for more extended observations. Initial results of this survey phase were discussed by Gilliland et al. (2010).

\section{Pulsating stars}

As a background for the discussion below of specific types of pulsating stars we provide a brief overview of the properties of stellar pulsations. For more detail, see Aerts et al. (2010). We restrict the discussion to slowly rotating stars and oscillations of modest amplitude. In this case the oscillations can, to leading order, be characterized as small perturbations around a spherically symmetric equilibrium structure; individual modes depend on co-latitude $\theta$ and longitude $\phi$ as spherical harmonics $Y_{l}^{m}(\theta, \phi)$, where $l$ 
measures the total number of nodal lines on the stellar surface and $m$ the number of nodal lines crossing the equator, with $|m| \leqslant l$. Modes with $l=0$ are typically described as radial oscillations. For each $l, m$ the star has a set of modes distinguished by the radial order $n$.

From a dynamic point of view there are two basic types of stellar modes $\dagger$ : acoustic modes (or p modes) where the restoring force is pressure, and internal gravity waves (or g modes) where the restoring force is buoyancy variations across spherical surfaces. Thus g modes are only found for $l>0$. Being acoustic the properties of the $\mathrm{p}$ modes predominantly depend on the sound-speed variation within the star. In many cases the result is that the frequencies $\nu$ approximately scale with the inverse dynamical time scale, or

$$
\nu \propto\left(\frac{G M}{R^{3}}\right)^{1 / 2} \propto\langle\rho\rangle^{1 / 2},
$$

where $M$ and $R$ are the mass and radius of the star, $G$ is the gravitational constant and $\langle\rho\rangle$ is the mean density. The g-mode frequencies depend on the variation of the gravitational acceleration and density gradient throughout the star, the latter in turn being very sensitive to the variation of composition.

In unevolved stars typical g-mode frequencies are lower than the frequencies of $\mathrm{p}$ modes. However, as the star evolves, the core contracts, leading to a very high local gravitational acceleration; in addition, strong composition gradients are built up by the nuclear burning and possibly convective mixing. In this case the local g-mode frequency may become large, and as a result the modes can take on a mixed character, with p-mode behaviour in the outer parts of the star and g-mode character in the deep interior. We discuss examples of this in Sections 7 and 8.

An important measure of the properties of a mode of oscillation is its normalized mode inertia

$$
E=\frac{\int_{V} \rho|\boldsymbol{\delta} \boldsymbol{r}|^{2} \mathrm{~d} V}{M\left|\boldsymbol{\delta} \boldsymbol{r}_{\mathrm{s}}\right|^{2}},
$$

where $\boldsymbol{\delta} \boldsymbol{r}$ is the displacement vector, $\boldsymbol{\delta} \boldsymbol{r}_{\mathrm{s}}$ the surface displacement, and the integral is over the volume $V$ of the star. Acoustic modes have their largest amplitude in the outer layers of the star and hence typically have relatively low inertias, whereas g modes are generally confined in the deep interiors of stars, with correspondingly high inertia.

Energetically, two fundamentally different mechanisms may excite the oscillations. Some stars function as a heat engine where the relative phase of compression and heating in a critical layer of the star is such that thermal energy is converted into mechanical energy, contributing to driving of the oscillations, and dominating over the other parts of the star which have the opposite effect. This is typically associated with opacity variations of specific elements; an important example is the effect of the second ionization of helium (Cox \& Whitney 1958), which causes instability in stars where the corresponding region in the star is located at the appropriate depth beneath the stellar surface. The driving leads to initially exponential growth of the oscillations, with so far poorly understood mechanisms setting in to control the limiting amplitudes of the modes.

In stars where the oscillations are not self-excited in this manner the modes may be excited stochastically, through driving from other dynamical phenomena in the star. This is likely the case in the Sun where near-surface convection at nearly sonic speed is a strong source of acoustic noise which excites the resonant modes of the star (e.g., Goldreich \&

$\dagger$ In addition, modes corresponding to surface gravity waves can be distinguished, but at degrees so far only relevant for spatially resolved observations of the Sun. 
Keeley 1977; Houdek et al. 1999). In this case the oscillation amplitudes result from a balance between the stochastic energy input and the damping of the modes. Such excitation is expected in all stars with a significant outer convection zone, i.e., stars with effective temperature below around $7000 \mathrm{~K}$. In principle, it excites all modes in a star; however, typically only modes with low inertia (cf. Eq. 3.2), i.e., acoustic modes of rather high radial order, are excited to sufficiently high amplitudes to be readily observable.

The stochastic excitation of acoustic oscillations leads to a characteristic bell-shaped distribution of mode amplitudes (see Fig. 9 below). It has been found (Brown et al. 1991; Brown \& Gilliland 1994; Kjeldsen \& Bedding 1995; Bedding \& Kjeldsen 2003; Stello

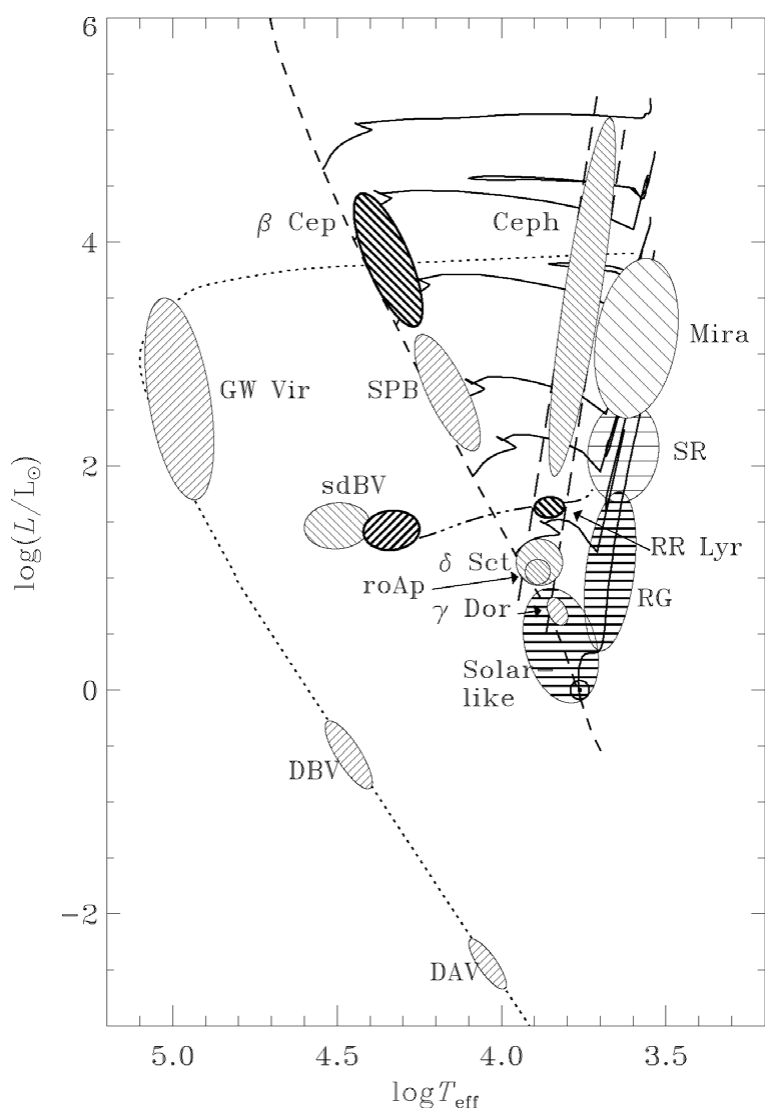

Figure 1. Schematic location of classes of pulsating stars in the Hertzsprung-Russell diagram. The diagonal dashed line marks the main sequence, where stars undergo central hydrogen burning. Evolution tracks following the main sequences for a few stellar masses are shown by solid lines, the triple-dot-dashed line indicates the location of horizontal-branch stars with central helium burning, and the dotted line sketches the white dwarf cooling track, after stars have stopped nuclear burning. The hatching indicates the excitation mechanism: slanted lines from lower right to upper left for heat-engine excitation of $\mathrm{p}$ modes, slanted lines from lower left to upper right for heat-engine excitation of g modes, and horizontal lines for stochastic excitation. The two nearly vertical dashed lines mark the limits of the Cepheid instability strip, where stars are generally excited by an opacity-driven heat engine operating in the second helium ionization zone. Stars discussed in the present paper are shown with bolder lines: RR Lyrae stars (RR Lyr; Section 4$)$, massive main-sequence stars ( $\beta$ Ceph; Section 5$)$, long-period subdwarf B variables (sdBV; Section 6), solar-like pulsators (Section 7) and red giants (RG; Section 8). 
RR Lyr ground-based data (2004)

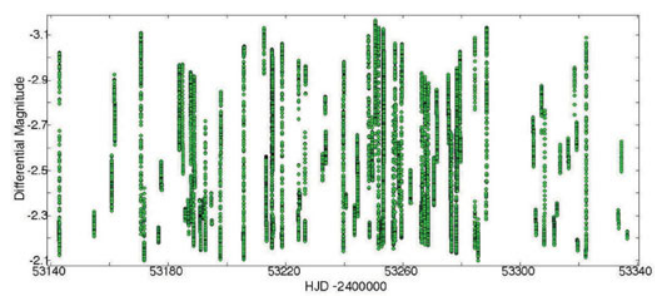

RR Lyr Kepler Q1+Q2 data (2009)

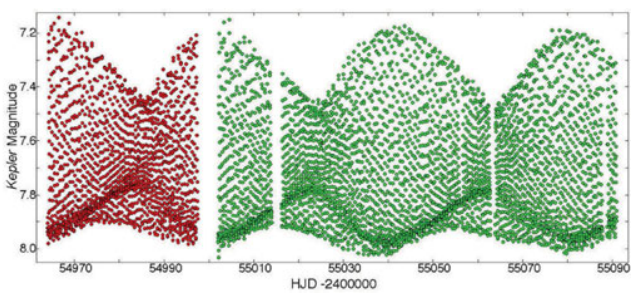

Figure 2. Lightcurves for RR Lyrae lightcurves. The left panel shows the combined results of observations with 6 ground-based telescopes (Kolenberg et al. 2006), compared with the first two quarters of Kepler data (Kolenberg et al. 2011).

et al. 2008) that the frequency $\nu_{\max }$ at maximum power scales as the acoustic cut-off frequency (Lamb 1909), leading to

$$
\nu_{\max } \propto M R^{-2} T_{\mathrm{eff}}^{-1 / 2},
$$

where $T_{\text {eff }}$ is the effective temperature. This relation so far lacks a solid theoretical underpinning (see, however Belkacem et al. 2011), but it has proven to be very useful in characterizing stars observed to have solar-like oscillations (see Section 8).

As illustrated in Fig. 1 pulsating stars are found throughout the Hertzsprung-Russell diagram, in all phases of stellar evolution. Thus there are excellent possibilities for learning about a broad range of stars. Most of these classes have been observed by CoRoT and Kepler. In the following we discuss a few important examples.

\section{RR Lyrae stars}

The RR Lyrae stars are amongst the 'classical' pulsating stars that have been studied extensively from the ground, since the discovery in 1900 of the variability of RR Lyr, the prototype of the class which happens to be in the Kepler field (see Smith 1995, for more information on these stars). They are low-mass stars with a low content of heavy elements, in the core helium-burning phase of evolution. As they have relatively welldefined luminosities they serve a very useful purpose as distance indicators to nearby galaxies, such as the Magellanic Clouds. Their pulsations are excited by the heat-engine mechanism, operating as a result of opacity variations in the second helium ionization zone. They oscillate predominantly in one or two low-order radial modes, with amplitudes large enough to allow observations with even very modest equipment.

What makes these stars interesting in the context of space asteroseismology are the very interesting, and poorly understood, dynamical properties of the oscillations in a substantial fraction of the class. Blažko (1907) $\dagger$ discovered in one member of the class that the maximum amplitude varied cyclically with a period of $40.8 \mathrm{~d}$. This phenomenon has since been found in a number of RR Lyrae stars, including RR Lyr itself (Kolenberg et al. 2006). A centennial review, including a discussion of the so far questionable attempts at explaining the effect, was provided by Kolenberg (2008).

The continuity, duration and precision of space-based observations offer obvious advantages in investigating such long-term phenomena. This is illustrated in Fig. 2 which

$\dagger$ In the discovery paper the original Cyrillic name was written as 'Blažko'. However, traditionally the name of the effect is now written as 'the Blazhko effect' with a slightly different transliteration; we follow that tradition here. 
compares results of an extensive ground-based campaign on RR Lyr with Kepler observations. The latter obviously provide a far better phase coverage throughout the Blazhko cycle. Phase plots at maximum and minimum amplitude in the cycle are illustrated in Fig. 3. Similar results have been obtained by CoRoT (Poretti et al. 2010). An early survey of 28 RR Lyrae stars by Kepler (Kolenberg et al. 2010) found the Blazhko effect in $40 \%$ of the stars, a rather higher fraction than previously suspected.

One may hope that these vastly improved data will bring us closer to an understanding of this enigmatic phenomenon. As an interesting piece of evidence Szabó et al. (2010), using Kepler data, found that three Blazhko RR Lyrae stars, including RR Lyr itself, showed period doubling in certain phases of the Blazhko cycle, with slight variations in the maximum amplitude between alternating pulsation cycles. Also, from CoRoT observations Chadid et al. (2011) investigated cycle-to-cycle changes in the Blazhko modulation. Such results evidently provide constraints on, and inspiration for, the attempts at theoretical modelling of these stars.

\section{Massive main-sequence stars}

The pulsations in hot main-sequence stars, the so-called $\beta$ Cephei stars, have been known for a century, but the cause of the pulsations was only definitely identified around 1990, when substantial revisions in opacity calculations produced opacities which allowed excitation of the observed modes through the heat-engine mechanism operating through the opacity from iron-group elements (Moskalik \& Dziembowski 1992). This causes
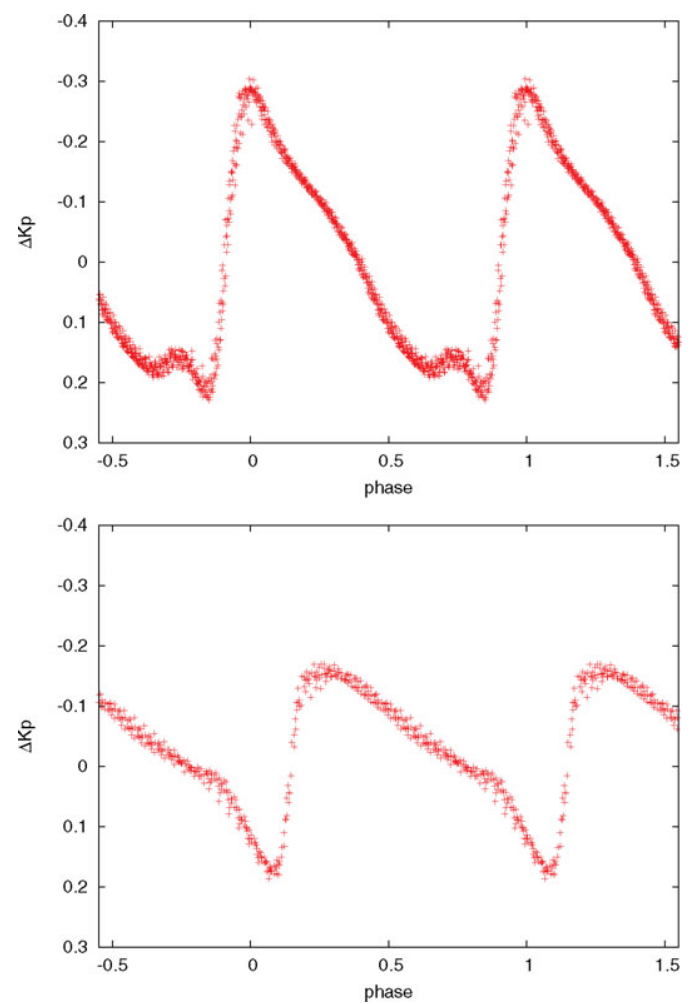

Figure 3. Phase plots of RR Lyr at maximum and minimum amplitude, from Kepler observations. Figure courtesy of R. Szabó. 
excitation of both $\mathrm{p}$ and $\mathrm{g}$ modes, with a tendency towards $\mathrm{g}$ modes at lower effective temperature and hence mass, and a transition to the slowly pulsating B stars (SPB stars) dominated by high-order g modes with very long periods. An excellent overview of the excitation of oscillations in the B stars was provided by Pamyatnykh (1999).

These massive stars are of very considerable astrophysical interest as precursors for the core-collapse supernovae. Their structure and evolution depend strongly on the properties of the convective core, including additional mixing outside the convectively unstable region caused by overshoot or 'semiconvection', as well as other dynamical phenomena associated, for example, with rotation. Thus the potential for asteroseismic investigation is very valuable, provided that adequate data can be obtained. Particularly useful diagnostics can be obtained from observations of g modes. High-order g modes are approximately uniformly spaced in period, with a period spacing $\Delta \Pi$ given, to leading order, by

$$
\Delta \Pi=\frac{2 \pi^{2}}{\sqrt{l(l+1)}}\left(\int_{r_{1}}^{r_{2}} N \frac{\mathrm{d} r}{r}\right)^{-1}
$$

(Tassoul 1980); here $N$ is the buoyancy frequency and $\left[r_{1}, r_{2}\right]$ is the interval where the modes are trapped, with $N^{2}>0$. Assuming an ideal gas the buoyancy frequency is determined by

$$
N^{2} \simeq \frac{g^{2} \rho}{p}\left(\nabla_{\mathrm{ad}}-\nabla+\nabla_{\mu}\right)
$$

where $g$ is the local gravitational acceleration, $\rho$ is density and $p$ is pressure; also, following the usual convention,

$$
\nabla=\frac{\mathrm{d} \ln T}{\mathrm{~d} \ln p}, \quad \nabla_{\mathrm{ad}}=\left(\frac{\partial \ln T}{\partial \ln p}\right)_{\mathrm{ad}}, \quad \nabla_{\mu}=\frac{\mathrm{d} \ln \mu}{\mathrm{d} \ln p},
$$

where $T$ is temperature, $\mu$ is the mean molecular weight and the derivative in $\nabla_{\text {ad }}$ is taken corresponding to an adiabatic change. In a detailed analysis Miglio et al. (2008) pointed out the diagnostic potential of departures from the uniform period spacing. Owing to the presence of the term in $\nabla_{\mu}$ in Eq. (5.2) the buoyancy frequency is very sensitive to the detailed composition profile, such as may result outside a convective core. The resulting sharp features in the buoyancy frequency introduce perturbations to $\Delta \Pi$ with a characteristic oscillatory behaviour, the details of which depend strongly on conditions at the edge of the core.

As for the RR Lyrae stars (Section 4) the oscillations can readily be detected in groundbased observations. The difficulty is to obtain adequate frequency resolution and precision, given the long periods and generally fairly dense spectra. Substantial successes have been achieved with coordinated multi-site observations over several months (Handler et al. 2004), leading to interesting information about convective core overshoot (Ausseloos et al. 2004; Pamyatnykh et al. 2004) and internal rotation (Aerts et al. 2003; Dziembowski \& Pamyatnykh 2008). However, it is evident that such massive campaigns can only be carried out in very special cases, and even then they do not provide the full desired data continuity or sensitivity to low-amplitude modes.

Observations from CoRoT and Kepler have the potential to secure very long continuous observations of these stars (Degroote et al. 2010a; Balona et al. 2011). A very interesting case was discussed by Degroote et al. (2010b), for the star HD 50230. This is a massive main-sequence star, of spectral type B3V, which was observed by CoRoT for 137 days. The resulting power spectrum showed a large number of g-mode frequencies, with periods up to a few days, in addition to several high-frequency p modes. In the long-period part 
of the spectrum the authors were able to identify a group of eight modes with almost constant period spacing, arguing that such a sequence is very unlikely to be found by chance. As illustrated in Fig. 4 these period spacings showed a highly regular variation with period, of precisely the form predicted by Miglio et al. (2008) to result from a sharp feature in the buoyancy frequency. As pointed out by Miglio et al. the decrease in the amplitude with increasing period is a sensitive diagnostic of the properties of the feature. A more detailed interpretation of the results will require more stringent characterization of other properties of the star, through 'classical' observations as well as more extensive asteroseismic analyses of the rich spectrum. However, the result clearly demonstrates the potential of such observations for characterizing the properties of convective cores in massive main-sequence stars.

\section{Subdwarf B stars}

The subdwarf B stars (sdB stars) are very hot core helium burning stars, at the blue end of the horizontal branch (for a review, see Heber 2009). The high effective temperature is the result of the stars having lost most of their hydrogen envelope, through processes that are so far not fully understood. Pulsations were first observed in such stars by Kilkenny et al. (1997), with very high frequency. That the stars might be unstable to acoustic modes was found in parallel, and independently, by Charpinet et al. (1996). The driving arises from the heat-engine mechanism operating on opacity from the irongroup elements. Subsequently Green et al. (2003) also observed long-period oscillations in somewhat cooler sdB stars, corresponding to g modes of high order. A detailed analysis of the excitation was carried out by Fontaine et al. (2003). To be effective, the irongroup opacity must be enhanced through enrichment of the elements in the critical region through radiative levitation (see also Fontaine et al. 2006); owing to the high gravitational acceleration such processes of settling and levitation are quite efficient in these stars.

As in the previous cases discussed, major ground-based efforts have been made to study these pulsations, involving coordinated observations between several observatories over extended periods (e.g., Randall et al. 2006a,b; Baran et al. 2009). The difficulties of such observations are particularly severe for the g-mode pulsators, with periods of order hours and amplitudes of only a few parts per thousand. Yet these modes are particularly

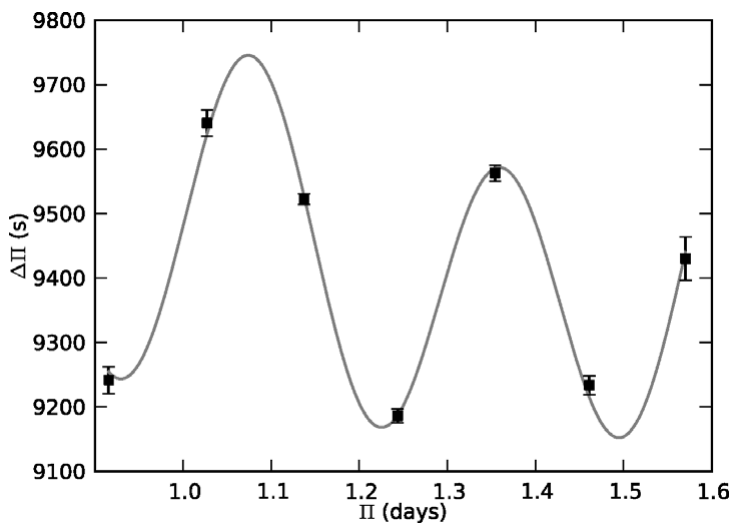

Figure 4. Period spacings in the B3V star HD 50230, from CoRoT observations. The variations in $\Delta \Pi$, here fitted with a decaying sinusoid, reflect the properties of the buoyancy frequency just outside the convective core in the star. Adapted from Degroote et al. (2010b). 
interesting as diagnostics of the deep interiors of the stars. Thus the sdB variables have been prime targets for asteroseismology with CoRoT and Kepler. The Kepler initial survey has included a substantial number of sdB stars and so far led to the discovery of 14 pulsating stars, all except one of which are long-period pulsators, with substantial numbers of $\mathrm{g}$ modes of intermediate and high order (Østensen et al. 2010, 2011; Baran et al. 2011). Thus the periods may be expected to satisfy the asymptotic relation (5.1). Reed et al. (2011) showed that this is indeed the case and noted the importance for mode identification.

As a specific example of the power of these data we consider a detailed analysis of the star KPD 1943+4058 based on the initial Kepler data. Here Reed et al. (2010) detected 21 modes, with periods between 0.7 and $2.5 \mathrm{~h}$, and in addition three modes in the p-mode region. Similar observational results were obtained by Van Grootel et al. (2010a), who in addition carried out a fit of the g-mode periods to models of the star. The models were described by their total mass, the mass in the thin envelope and an assumed fully mixed core, and the composition of the core, characterized by the combined abundance of carbon and oxygen. In addition, the models were constrained to be consistent with the spectroscopically inferred effective temperature and surface gravity. The fit to the observations measured by a merit function $S$ defined by

$$
S^{2}=\sum_{i}\left(\Pi_{i}^{(\mathrm{obs})}-\Pi_{i}^{(\mathrm{mod})}\right)^{2}
$$

where $\Pi_{i}^{(\text {obs })}$ and $\Pi_{i}^{(\mathrm{mod})}$ are the observed and model periods, respectively, and the identification of the computed modes with the observed modes is part of the fitting procedure. Some results are illustrated in Fig. 5. The analysis provided very precise determinations of the properties of the star, including strong evidence for a mixed core region significantly larger than the expected convectively unstable region, indicating substantial core overshoot.

It should be noted that the periods of the best-fitting model did not agree with the observed periods to within their observational uncertainty. This evidently shows that further improvements of the modelling, beyond the parameters included in the fit, are needed. It seems likely that the number of observed periods is sufficient that a formal
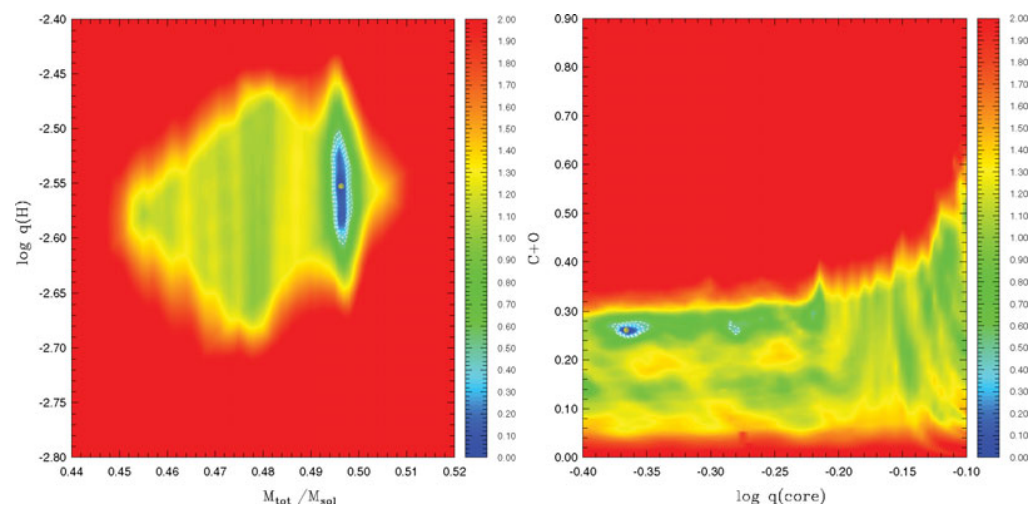

Figure 5. The merit function $S^{2}$ (cf. Eq. 6.1) in a fit of the observed Kepler periods for the star KPD $1943+4058$ to a set of models; the colour scale for $\log S^{2}$ is indicated to the right. The left panel shows the fit in terms of the total mass of the star, in solar units, and the logarithm of the fraction of the mass in the hydrogen-rich envelope. The right panel plots the merit as a function of the logarithm of the mass fraction outside the mixed core and the combined abundance, by mass, of carbon and oxygen in the core. From Van Grootel et al. (2010a). 
inversion of the differences can be attempted, as has been applied with great success in the solar case (e.g., Gough et al. 1996). The results may provide a direct indication of the aspects of the models where improvements are needed.

Similar analyses will be possible for the remaining stars for which extensive g-mode data have been obtained with Kepler, and they will evidently improve as the stars continue to be observed. Also, an sdB star showing extensive g-mode pulsations has been observed by the CoRoT mission (Charpinet et al. 2010). Model fitting to the resulting periods by Van Grootel et al. (2010b) yielded results rather similar to those discussed above.

\section{Solar-like oscillations in main-sequence stars}

Solar-like oscillations are predominantly acoustic in nature and excited by turbulent convection in the star's outer convective envelope. As already noted, although this broadband excitation mechanism excites all modes in principle, because of their low mode inertias it tends to be the high-order $\mathrm{p}$ modes that are excited to observable amplitude. The first star in which such oscillations were detected was of course the Sun, and the study of the Sun's oscillations has led to the rich field of helioseismology, in which Juri Toomre has played a leading role (see, e.g., Gough \& Toomre 1991; Christensen-Dalsgaard 2002).

The asteroseismic investigation of solar-type stars has taken a major step forward thanks to the Kepler mission (Chaplin et al. 2010). To date, Kepler has yielded clear detections of oscillations in 500 solar-type stars (Chaplin et al. 2011). A plot of the distribution in the HR diagram of Kepler stars with detected solar-like oscillations is shown in Fig. 6. This represents an increase by more than a factor of 20 of the number of known stars on and near the main sequence which show solar-like oscillations.

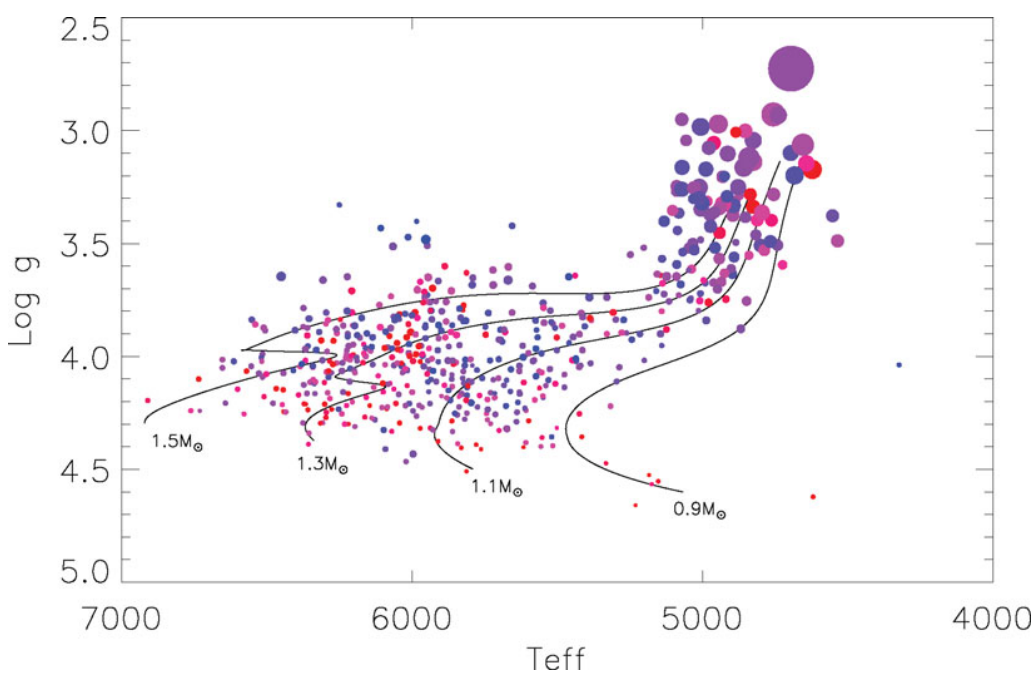

Figure 6. Location of stars observed by Kepler to show solar-like oscillations, plotted as a function of effective temperature and the logarithm of the surface gravity. The size of the symbols measures the oscillation amplitude, while the colour (in the electronic version) indicates the apparent brightness of the stars, with red for the brightest stars. For comparison, evolution tracks at the indicated masses are also shown. Adapted from Chaplin et al. (2011) and Verner et al. (2011). Figure courtesy of C. Karoff. 
The high-order, low-degree p modes in solar-type stars occupy resonant acoustic cavities that extend from the surface to a region close to the stellar core. Their cyclic frequencies $\nu_{n l}$ satisfy a simple expression:

$$
\nu_{n l} \simeq \Delta \nu\left(n+\frac{l}{2}+\epsilon\right)-d_{n l} .
$$

Here

$$
\Delta \nu=\left(2 \int_{0}^{R} \frac{\mathrm{d} r}{c}\right)^{-1}
$$

where $c$ is the adiabatic sound speed and the integral is over the distance $r$ to the centre of the star; also

$$
d_{n l}=\frac{l(l+1) \Delta \nu}{4 \pi^{2} \nu_{n l}}\left[\frac{c(R)}{R}-\int_{0}^{R} \frac{\mathrm{d} c}{\mathrm{~d} r} \frac{\mathrm{d} r}{r}\right]
$$

(Tassoul 1980; Gough 1986). Accordingly, the frequencies of such modes of the same degree are separated by large separations

$$
\Delta \nu_{n l}=\nu_{n l}-\nu_{n l-1} \approx \Delta \nu,
$$

while the small correction $d_{n l}$ gives rise to small separations

$$
\delta \nu_{n l}=\nu_{n l}-\nu_{n-1 l+2} \approx(4 l+6) \frac{\Delta \nu}{4 \pi^{2} \nu_{n l}}\left[\frac{c(R)}{R}-\int_{0}^{R} \frac{\mathrm{d} c}{\mathrm{~d} r} \frac{\mathrm{d} r}{r}\right]
$$

between the frequencies of modes that differ in degree by 2 and in order by 1 . Finally $\epsilon$ is a slowly varying function of frequency which is predominantly determined by the properties of the near-surface region.

The quantity $\Delta \nu$ is a measure of the acoustic radius of the star. It shares the scaling (Eq. 3.1) of the frequencies with the mean density, and hence so too do the large separations. For main-sequence stars $d_{n l}$, and thus the small separations, are mainly determined by the central regions of the star, being sensitive in particular to the sound-speed gradient in the core, and hence they provide a measure of the star's evolutionary state. Thus measuring the large and small frequency separations gives a measure of the mean density and evolutionary state of the star. A useful seismic diagnostic is the asteroseismic HR diagram, in which the star's average large separation is plotted against its average small separation: this is illustrated in Fig. 7. For main-sequence stars, the asteroseismic HR diagram allows the mass and age of the star to be estimated, assuming that other physical inputs (such as initial chemical composition and the convective mixing-length parameter) are known.

The existence of the large separation also motivates another diagnostic, which is to plot the frequencies of the star in a so-called échelle diagram. Here the frequencies $\nu_{n l}$ are reduced modulo $\Delta \nu$ and $\bar{\nu}_{n l} \equiv \nu_{n l} \bmod \Delta \nu$ is plotted on the x-axis while $\nu_{n l}-\bar{\nu}_{n l}$ is plotted on the $y$-axis. If the spacing of frequencies according to asymptotic expression (7.1) were exact, the modes of like degree would be aligned as nearly vertical lines in the échelle diagram, with the lines corresponding to $l=0,2$ being separated from one another by the small separation and the line corresponding to $l=1$ being offset from those by an amount corresponding to half the large separation. Deviations from such a simple picture reveal deviations from the simple asymptotic relation and contain physically interesting information about the star. An example of an échelle diagram for star KIC 11026764, is shown in Fig. 8. The ridges corresponding to $l=0,2$ are evident. The ridge corresponding 


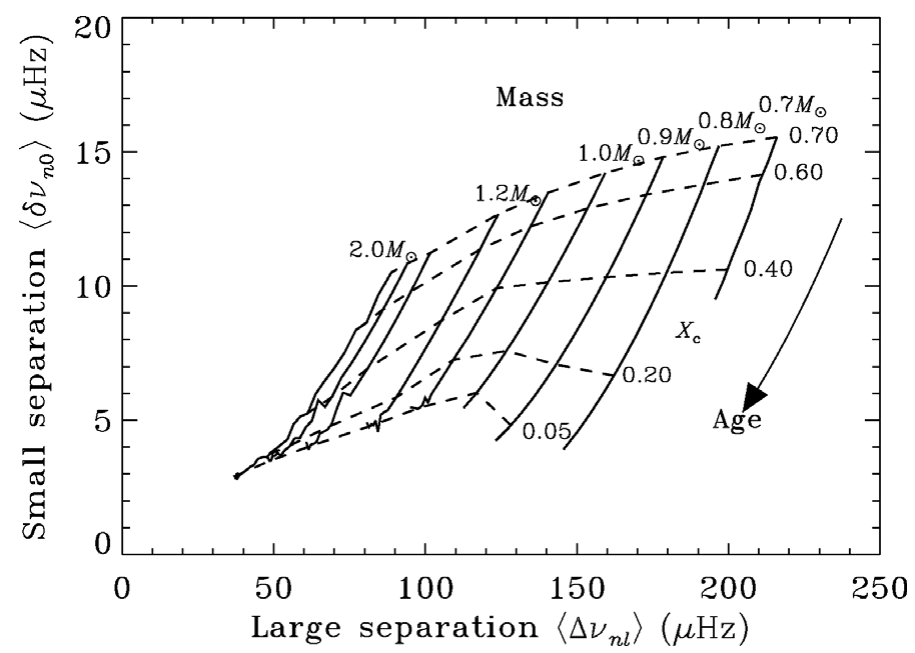

Figure 7. Asteroseismic HR diagram for a homogeneous set of stellar models of different masses and ages. The solid lines show how a star of given mass evolves in terms of its large and small frequency separations. The dashed lines connect stars which have aged by the same fraction of their total main-sequence lifetime.

to $l=1$ is more irregular: this is due to avoided crossings in this relatively evolved star, an issue which is discussed further below.

Even without a measurement of the small separations, it is possible to make useful seismic estimates of stellar masses and radii using observational measures of $\Delta \nu$ and of the frequency $\nu_{\max }$ of maximum mode amplitude. From Eq. (7.2) the former scales as $M / R^{3}$, whereas by Eq. (3.3) the latter scales as $M / R^{2}$ : hence a measurement of these two yields an estimate of both $M$ and $R$. This has been applied to an ensemble of 500 stars in the Kepler field by Chaplin et al. (2011). This paper by Working Group 1 (WG1) of the KASC concludes that while the estimated radii of the 500 stars are similar to those expected from stellar population models, the observed distribution of masses is wider at its peak than the model distribution, and is offset towards slightly lower masses.

Chaplin et al. (2010) published observations of three bright solar-type stars, which were monitored during the first month of Kepler science operations. This paper was the first to establish the asteroseismic potential of Kepler observations of solar-type stars: about 20 modes were distinguished in each star, and the frequencies and frequency separations allowed radii, masses and ages of the stars to be estimated. The three stars that were the objects of the study, KIC 6603624, KIC 3656476 and KIC 11026764, were given the working names Saxo, Java and Gemmat by the WG1 members. One of these stars, Gemma, was revealed to have evolved off the main sequence and proved more challenging to model and constrain asteroseismologically: this interesting case is discussed further now.

Gemma is one of the best-studied solar-like stars to be investigated thus far with Kepler data. The observed power spectrum, as obtained by Chaplin et al. (2010), is shown in Fig. 9. The analysis of the frequencies of the star was the subject of the paper by Metcalfe et al. (2010). Gemma is 10-20 per cent more massive than the Sun and also somewhat older. The core of Gemma is therefore more chemically evolved than is the core of the

$\dagger$ The working group allocated the names of pet cats to the stars that have been the early objects of study: this ideosyncracy is due to the WG1 lead, Bill Chaplin. 
Sun; the models indicate that the star has a small compact helium core, surrounded by a hydrogen-burning shell. This leads to interesting behaviour of the star's frequencies which provides a powerful diagnostic of the star's evolutionary state. As a solar-like star evolves at the end of its main-sequence life, it continues to grow in radius while forming a strong gradient in mean-molecular weight at the edge of its core. Also, the core contracts, increasing the central condensation and the gravitational acceleration in the deep interior of the star. These effects in turn cause a strong peak to form in the buoyancy frequency (cf. Eq. 5.2), which supports g modes at frequencies which are representative for the stochastically excited solar-like oscillations. Thus at such frequencies the star has two resonant cavities supporting nonradial modes: one in the deep envelope of the star where the modes behave like $\mathrm{p}$ modes, increasingly confined to the outer regions of the star with increasing degree, and one in the core where the modes behave like g modes. These two regions are separated by an intermediate region where the modes are evanescent.

With increasing age the star undergoes an overall expansion which causes the frequencies of the p modes to decrease, while the increase in the central condensation and hence the buoyancy frequency causes the frequencies of $g$ modes to increase. Although the $g$ modes are not in general themselves observable directly, at times in the star's evolution the frequencies of a $\mathrm{g}$ mode and a $\mathrm{p}$ mode get sufficiently close for a strong coupling between the two modes to be possible, giving rise to a so-called mixed mode. This evolution of the frequency spectrum is illustrated in Fig. 10 for a representative stellar model of Gemma. This shows how the radial $(l=0)$ and $l=1 \mathrm{p}$-mode frequencies change as a function of age of the star. By their nature, g modes are non-radial; and hence the radial modes cannot couple to them: thus the overall expansion of the star simply causes the $l=0$ frequencies to decrease monotonically with increasing age. The $l=1$ frequencies also tend to decrease with age; but occasionally a given $l=1$ mode approaches the frequency of an $l=1 \mathrm{~g}$ mode: at that point, a strong coupling between the two modes occurs if the evanescent region between their two resonant cavities is not too large. The frequencies of the two modes never actually cross: instead, the modes undergo an avoided crossing, which results in the observable mode increasing in frequency as the star evolves,

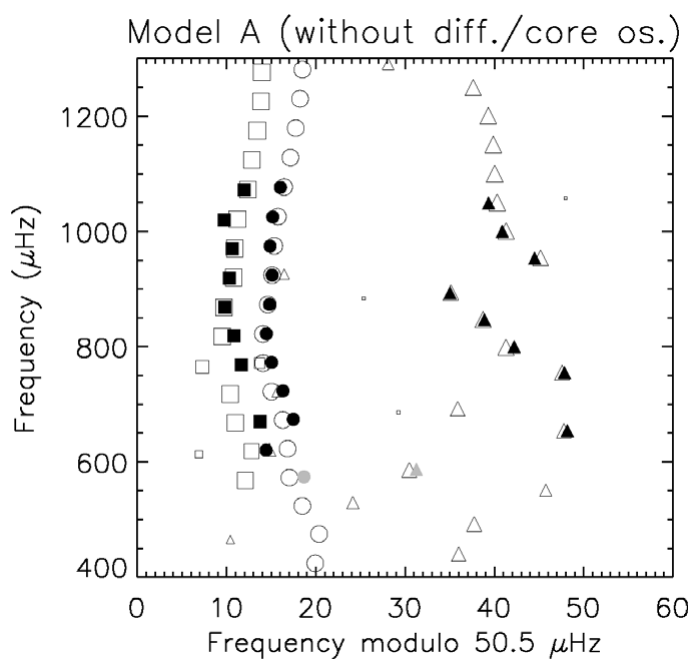

Figure 8. An échelle diagram for the solar-type star KIC 11026764 (Gemma) indicating both the observed frequencies (filled symbols) and those of a stellar model (open symbols). Modes of degree $l=0,1,2$ are denoted respectively by circles, triangles and squares. From Metcalfe et al. (2010). 
for the duration of the strong mode coupling. The evolving frequencies of the physical g modes can be discerned in Fig. 10 as the loci of the avoided crossings that take place for the $l=1 \mathrm{p}$ modes.

The frequency spectrum of $l=0$ and $l=1$ modes at any particular stellar age can be read off Fig. 10 by taking a vertical cut through the ridges: an example at an age of about $5.98 \mathrm{Gyr}$ is indicated. It is evident that the $l=0$ modes will be essentially evenly spaced in frequency, consistent with the asymptotic expression (Eq. 7.1), whereas the series of avoided crossings will cause the $l=1$ modes to be nonuniform. Moreover, the location in frequency space where avoided crossings are "caught in the act" is strongly dependent on the age of the star and so can enable the age of the star to be determined rather precisely.

This behaviour is consistent with measured frequencies of Gemma, illustrated in the échelle diagram in Fig. 8. The $l=0$ and $l=2$ frequencies are approximately uniformly spaced, whereas the $l=1$ frequencies are more irregularly spaced, consistent with avoided crossings. (The $l=2$ and higher-degree p modes are affected much less by coupling to the $\mathrm{g}$ modes than are the $l=1$ modes, because their lower turning points are further from the core and hence the evanescent region between the resonant cavities of the $\mathrm{p}$ and g modes is wider.)

Metcalfe et al. (2010) modelled Gemma, fitting to the individual measured frequencies and hence exploiting in particular the non-uniform distribution of the $l=1$ modes. The frequencies of one of their resulting models are shown in the échelle diagram. They found that two families of solutions, one with stellar masses around $1.1 M_{\odot}$ and the other with stellar masses around $1.2 M_{\odot}$, that fitted the observed frequencies equally well. Notwithstanding this $10 \%$ ambiguity in mass, the radius and age of the star were determined with a precision of about $1 \%$, and an estimated accuracy of about $2 \%$ for the radius and about $15 \%$ for the age. The mass ambiguity would be resolved if the range of

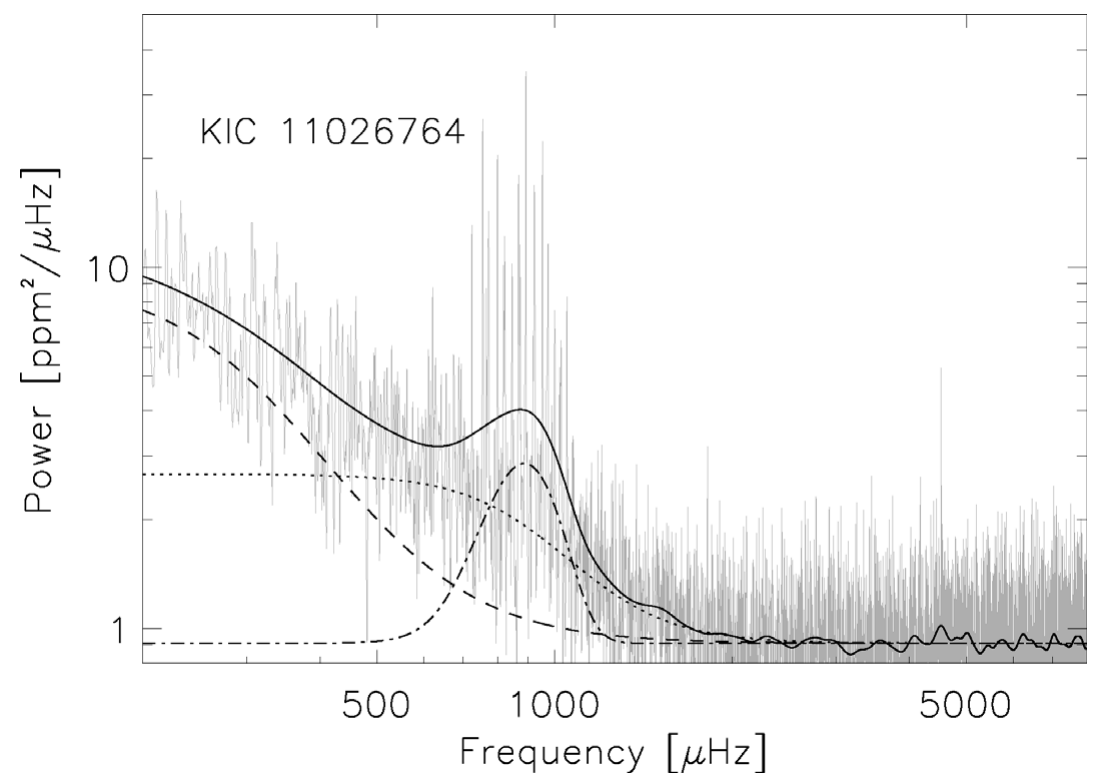

Figure 9. Observed power spectrum of the star KIC 11026764, known as Gemma, based on early Kepler observations. The solid line shows the smoothed spectrum, separated into the oscillation signal (dash-dotted line) and background components from granulation and faculae (dashed and dotted lines, respectively). Figure, adapted from Chaplin et al. (2010), courtesy of C. Karoff. 
measured frequencies could be extended to higher frequencies, at which point the model frequencies not only of the $l=1$ modes but also of the $l=0,2$ modes diverge between the two families of models.

The properties of the modes in the vicinity of the avoided crossings is illustrated in more detail in terms of the mode inertia (cf. Eq. 3.2) in Fig. 11. When the dipolar modes behave predominantly as acoustic modes, with frequencies decreasing with increasing age, their inertia is close to that of the neighbouring radial mode. It increases when a mode behaves predominantly as a g mode; at the point of closest approach in an avoided crossing the two modes have the same inertia, intermediate between the g-mode and the p-mode behaviour. As discussed below for red giants, the inertia has an important influence on the mode visibility. For the dipolar modes in the Gemma models the contrast between the p- and g-mode behaviour is modest and the modes are readily observable, even when they are most g-mode-like. On the other hand, for modes of degree $l=2$ and higher the intermediate evanescent region is substantially broader and the distinction between the p- and g-mode behaviour correspondingly stronger. Thus it is less likely to observed mixed modes at these higher degrees, as confirmed by the interpretation of the observed spectrum.

Apart from the intrinsic interest in the asteroseismic studies of solar-like stars, these studies provide an important possibility for characterizing stars that host extra-solar planetary systems. When a planet is detected using the transit technique the variation in the detected stellar brightness depends on the ratio between the diameters of the planet and the central star. A reliable determination of the planet radius, of great importance to the characterization of the nature of the planet, therefore depends on determining the stellar radius. As discussed above this can be provided by the asteroseismic analysis of the oscillations of the star; indeed, the target stars for the Kepler search for extra-solar planets are generally in a range of parameters where solar-like oscillations are expected, although most stars are too faint for reliable observations to be possible. However, the potential of the technique was demonstrated by Christensen-Dalsgaard et al. (2010) who analysed Kepler asteroseismic data for a known exoplanet host. More recently, astero-

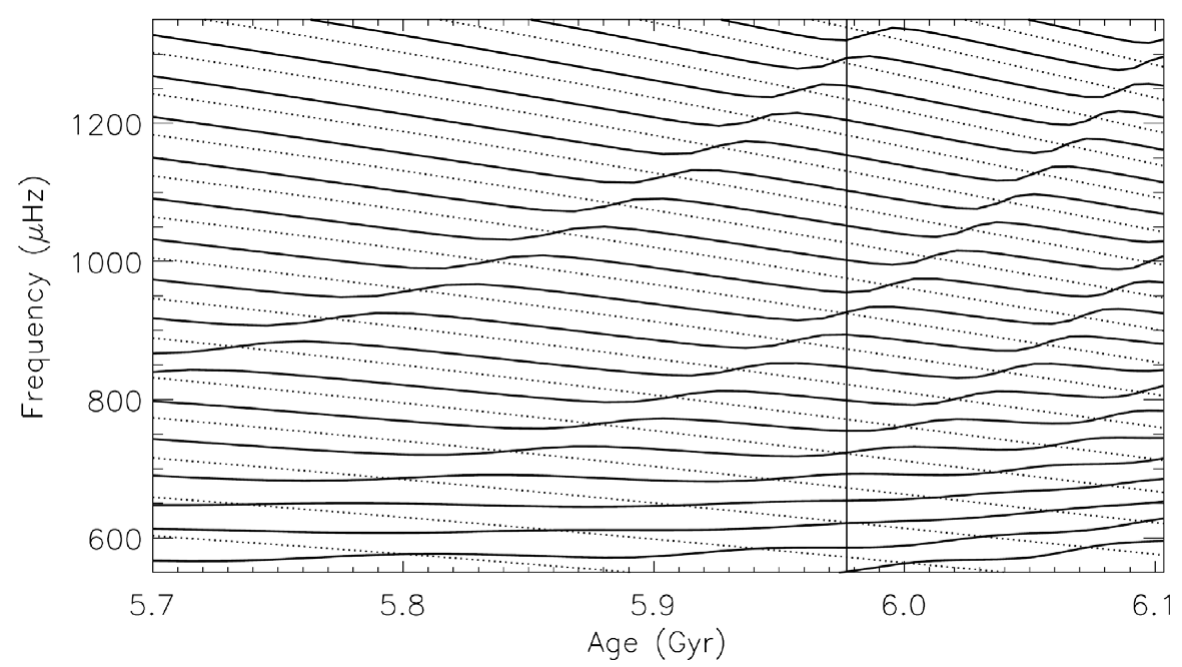

Figure 10. Evolution of the $l=0$ (dotted) and $l=1$ (solid) mode frequencies as a function of age for a representative stellar model of KIC 11026764 (Gemma). The vertical line indicates the age of 5.77 Gyr of one good-fitting model to Gemma's observed frequencies. From Metcalfe et al. (2010). 
seismic analysis was used in the characterization of the first rocky planet detected by Kepler (Batalha et al. 2011).

We finally note that the frequencies of solar-like oscillation are sensitive to stellar magnetic activity. In the solar case this has been studied extensively (e.g., Woodard \& Noyes 1985; Libbrecht \& Woodard 1990). Some evidence for such variation was found by Metcalfe et al. (2007) for the star $\beta$ Hyi. As discussed by Karoff et al. (2009) the long observing sequences possible with CoRoT and in particular Kepler provide a rich possibility for detecting similar effects in other stars. In fact, in a solar-like star observed by CoRoT García et al. (2010) detected variations in oscillation frequencies and amplitudes which appeared to be the result of a rather short stellar activity cycle.

\section{Red giants}

Assuming that the solar oscillations are excited stochastically by convection (Goldreich \& Keeley 1977) one would expect that all stars with vigorous outer convection zones exhibit such oscillations. A rough estimate of the amplitudes (Christensen-Dalsgaard \& Frandsen 1983) suggested that the amplitude increases with increasing luminosity. Thus red giants are obvious targets for the search for, and investigation of, solar-like oscillations.

The first definite detection of individual modes in a red giant was obtained by Frandsen et al. (2002) in the star $\xi$ Hya. The frequency spectrum showed very clearly a series of
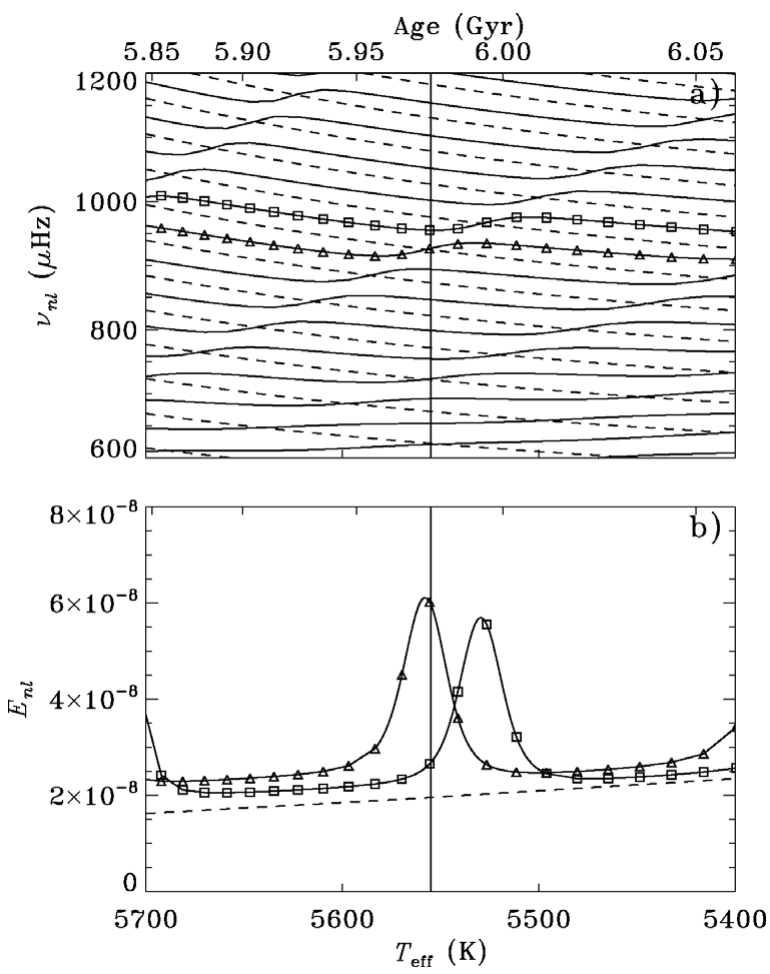

Figure 11. Panel a) shows a blow-up of Fig. 10 around the model illustrated in Fig. 8. Panel b) shows mode inertias for two of the $l=1$ modes (solid lines, identified in both panels by triangles and squares, respectively), and the intervening radial mode (dashed line). The increase in the inertia, relative to the radial mode, is an indication of a predominant g-mode character of the modes. 
uniformly spaced peaks with a separation $\Delta \nu \simeq 7 \mu \mathrm{Hz}$. Simple modelling, given also the location in the HR diagram, strongly suggested that only radial modes had been observed; the alternative identification, of alternating $l=0$ and $l=1$ modes, would correspond to a true large separation twice as big and hence a radius smaller by roughly a factor 1.3, entirely inconsistent with the observed luminosity and effective temperature.

Evidence for solar-like oscillations in giants had also been obtained from more statistical analyses. Christensen-Dalsgaard et al. (2001) noted that the relation between the standard deviation and mean of the amplitude variations in the so-called semi-regular variables, based on visual observations carried out by the American Association of Variable Star Observers (AAVSO), was consistent with the expectations for stochastically excited oscillations. The solar-like nature of the oscillations of selected semi-regular variables was confirmed by Bedding (2003) through analysis of their power spectra. Also, Kiss \& Bedding $(2003,2004)$ analysed large sets of OGLE $\dagger$ observations of red giants, obtaining clear indication of several modes of oscillation which may likely be identified as solar-like. Detailed analyses of the OGLE data were carried out by Soszyński et al. (2007) and Dziembowski \& Soszyński (2010), confirming the solar-like nature of the observed oscillations. These investigations extend the realm of solar-like oscillations to stars with a luminosity of up to $10000 L_{\odot}$ and periods of several months.

The red-giant phase (see Salaris et al. 2002, for a review) follows after the phase exemplified by Gemma, discussed in Section 7 above. The stars ascend the Hayashi region at almost constant effective temperature and strongly increasing radius and luminosity, with a very compact helium core and an extended, mostly convective, envelope. The energy production takes place through hydrogen fusion in a thin shell around the helium core. The tip of the red-giant branch is defined by the ignition of helium near the centre. Stars with central helium fusion are located in the so-called 'red clump' in the HR diagram (see Fig. 15); even for these, however, most of the energy is produced by the hydrogen shell. The strongly centralized helium fusion gives rise to a small convective core, although the bulk of the helium core remains radiative. In both the ascending red giant and the clump phase the small extent of the core gives rise to a very high gravitational acceleration and hence buoyance frequency, further amplified by the presence of strong composition gradients (cf. Eq. 5.2). Thus all nonradial modes have the character of high-order g modes in the core. The resulting mixed nature of the modes, and the high density of modes of predominantly g-mode character, are illustrated in Fig. 12 which shows the mode inertia $E$ (cf. Eq. 3.2) for a typical model on the ascending red-giant branch. Most of the modes with $l=1$ and 2 clearly have much higher inertias than the radial modes, and hence are predominantly g modes. However, there are resonances where the modes are largely trapped in the outer acoustic cavity. These p-dominated modes have inertias close to the inertia of the radial modes, reflecting their small amplitudes in the core.

Dziembowski et al. (2001) considered the excitation and damping of modes in red giants. They found that the very high radial order of the nonradial modes in the core led to substantial radiative damping, even for modes that were predominantly acoustic. On this basis Christensen-Dalsgaard (2004) concluded that nonradial oscillations were unlikely to be observed in red giants. (This would be consistent with the results of Frandsen et al. (2002) on $\xi$ Hya where apparently only radial modes were found.) Fortunately this conclusion was wrong: nonradial modes are indeed observed in red giants and provide fascinating diagnostics of their interiors.

A first indication of the presence of nonradial oscillations in red giants came from line-profile observations by Hekker et al. (2006). However, the major breakthrough came

$\dagger$ Optical Gravitational Lensing Experiment 
with the observation of a substantial number of red giants by CoRoT, as presented by De Ridder et al. (2009). A selection of the resulting power spectra are shown in Fig. 13. The presence of solar-like oscillations is obvious, the peaks shifting to lower frequency with increasing radius (cf. Eq. 3.3). Also, De Ridder et al. (2009) showed an example of an échelle diagram which beyond any doubt identified modes of degree 0,1 and 2 .

The potential visibility of nonradial modes in red giants was made very much clearer by Dupret et al. (2009), following an analysis by Chaplin et al. (2005). The observational visibility of a mode is determined by the peak height $H$ in the power spectrum. This is related to the total observed power $P$ of the mode by $P \propto H \Delta$, where $\Delta$ is the width of the peak. If the mode is observed for much longer than the natural damping time, the width is given by the damping rate, i.e., the imaginary part $\left|\omega_{i}\right|$ of the frequency. If the damping is dominated by the near-surface layers, as is often the case, at a given frequency $\omega_{\mathrm{i}}$ is related to the mode inertia $E$ by

$$
\omega_{\mathrm{i}} \propto E^{-1} .
$$

Thus those modes that are predominantly g modes, with high inertia (cf. Fig. 12) have much smaller widths than the p-dominated modes. The power in the mode is determined by a balance between the energy input from the stochastic noise near the stellar surface and the damping. Assuming again Eq. (8.1) the outcome is that $P \propto E^{-1}$ at fixed frequency. It follows that the peak height $H$ is independent of $E$ at a given frequency and hence that the g-dominated modes should be observed at the same height as the p-dominated modes.

This, however, assumes that the duration $\mathcal{T}$ of the observation is much longer than the lifetime $\left|\omega_{\mathrm{i}}\right|^{-1}$ of the mode. If this is not the case, the peaks are broader and the height consequently smaller. As an approximate scaling of this dependence Fletcher et al. (2006) proposed

$$
H \propto \frac{P}{\left|\omega_{\mathrm{i}}\right|+2 / \mathcal{T}} ;
$$

for $\mathcal{T} \ll\left|\omega_{\mathrm{i}}\right|^{-1}$, in particular, $H \propto P \propto E^{-1}$ and the g-dominated modes are essentially

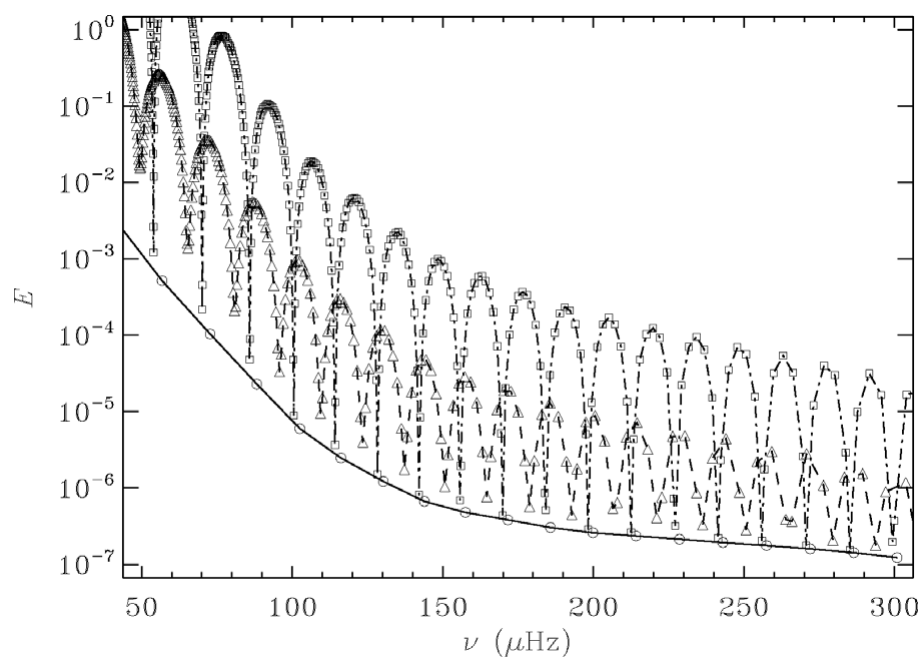

Figure 12. Mode inertias (cf. Eq. 3.2) against cyclic frequency in a red-giant model of mass $1.4 M_{\odot}$ and radius $5 R_{\odot}$. Modes of degree $l=0$ (circles connected by a solid line), $l=1$ (triangles connected by a dashed line) and $l=2$ (squares connected by a dot-dashed line) are illustrated. 
invisible. As is clear from Fig. 12 the g-dominated modes in practice often have inertias much higher than the p-dominated modes and hence correspondingly longer lifetimes; thus they may be expected have small observed peak heights, unless observations of very long duration are analysed. However, modes of mixed character, particularly those with $l=1$, may have damping times comparable with or shorter than the very long observations made available by CoRoT and Kepler and hence may be visible. Concerning $\xi$ Hya, the apparent absence of nonradial modes in the observations was probably caused by the relatively short observing run of around one month, compared with the five-month observations by De Ridder et al. (2009). Even the most p-mode-like dipolar modes have somewhat higher mode inertia and hence lifetimes than the radial modes; thus the peak height of these modes was likely suppressed in the observations by Frandsen et al. (2002).

Very extensive results on red-giant oscillations have been obtained by CoRoT and Kepler (e.g., Hekker et al. 2009; Bedding et al. 2010; Mosser et al. 2010; Kallinger et al. 2010a,b; Stello et al. 2010; Hekker et al. 2011). These confirm the acoustic nature of the observed spectra, with a clear detection of modes of degree 0,1 and 2 . This is illustrated in Fig. 14 (see also Gilliland et al. 2010), for a large sample of stars observed with Kepler during the first 16 months of the mission; the observations are shown as stacked spectra ordered according to decreasing large frequency separation $\Delta \nu$ and hence increasing radius and luminosity. This is characterized at the right-hand edge of the figure by the ratio between luminosity and mass, estimated from the oscillation parameters (see below). Stellar 'noise' from granulation is evident in the low-frequency region. The frequencies

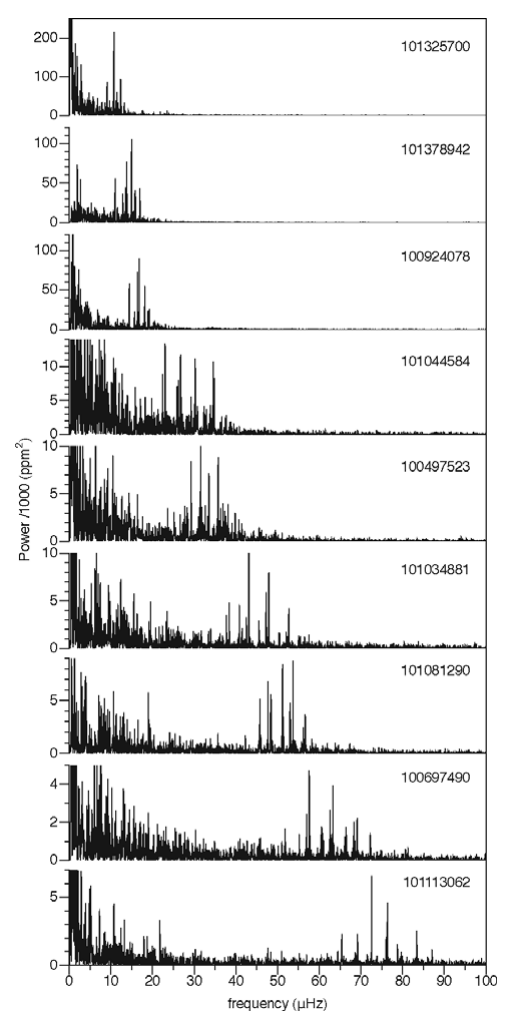

Figure 13. Power spectra of solar-like oscillations in red giants, from five months of observations with CoRoT. The stars are identified by their CoRoT identification number, with radius increasing towards the top. From De Ridder et al. (2009). 
approximately satisfy an asymptotic relation similar to Eq. (7.1), with a closely spaced pair of bands of $l=0,2$ modes and an intermediate band of $l=1$ modes. However, since the acoustic propagation region is generally confined to the convection zone or the region just beneath it the small separation between $l=0$ and 2 is not directly related to the properties of the stellar core, let alone the age of the star, unlike the situation on the main sequence. Montalbán et al. (2010a) carried out an extensive analysis of the overall properties of the oscillation frequencies for a large sample of red-giant models. They noted that the outer convective zone in red-clump phase is not quite as deep as for the stars ascending the red-giant branch; the extent of the acoustic propagation region beyond the convective envelope was found to have a potentially measurable effect on the small frequency separations.

The mean large frequency separation $\Delta \nu$ and the frequency $\nu_{\max }$ at maximum power satisfy the scaling relations (3.1) and (3.3). Thus the stellar properties can be characterized by these quantities. This is used in Fig. 15 to plot a 'Hertzsprung-Russell' diagram of red giants observed with Kepler, replacing the luminosity by $\nu_{\max }$ as a measure of radius and hence luminosity. The observations are compared with evolution tracks for a range of masses, using scaling from the solar value of $\nu_{\max }$. The distribution of stars clearly shows the higher density in the region of the helium-burning red clump. The scaling relations can also be used to determine the stellar parameters from the observed $\Delta \nu, \nu_{\max }$ and $T_{\text {eff }}$ (e.g. Kallinger et al. 2010a). This provides a unique possibility for population studies of red giants (e.g., Miglio et al. 2009; Kallinger et al. 2010b; Mosser et al. 2010; Hekker et al. 2011). The CoRoT results are particularly interesting in this regard, given that they allow a comparison of the populations in the centre and anti-centre directions of the Galaxy (Miglio et al., in preparation) and hence provide information about the evolution and dynamics of the Galaxy. A more precise determination of the stellar parameters can be obtained with the so-called grid-based methods, where stellar modelling is used to relate the effective temperature, mass and radius (e.g., Gai et al. 2011). This was used by Basu et al. (2011) to investigate the properties of two open clusters in the Kepler field.

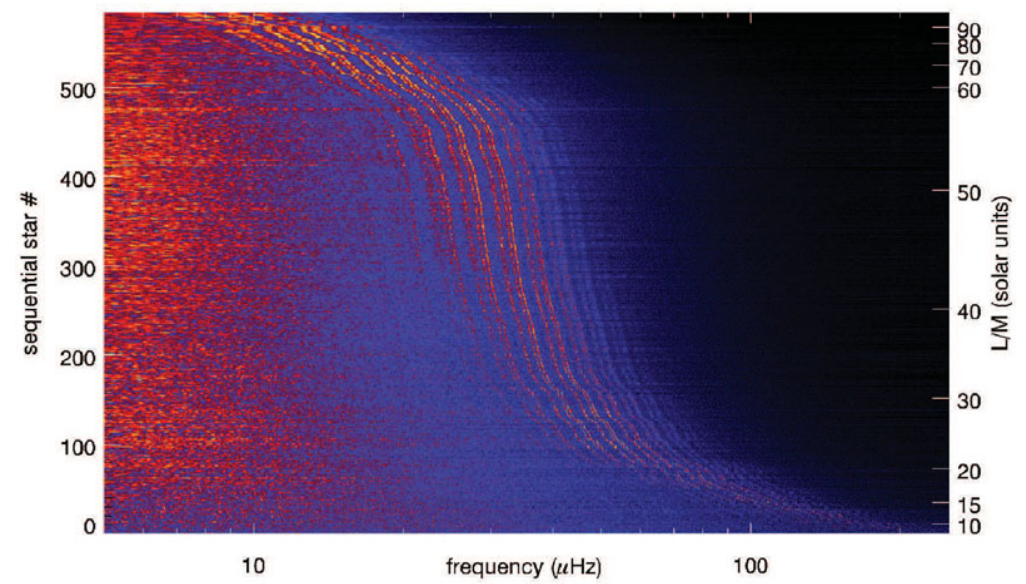

Figure 14. Stacked power spectra of red giants observed with Kepler, with the frequency of maximum power decreasing towards the top. The numbers at the right edge provide an estimate of the corresponding ratio between luminosity and mass, in solar units. Figure courtesy of T. Kallinger. 


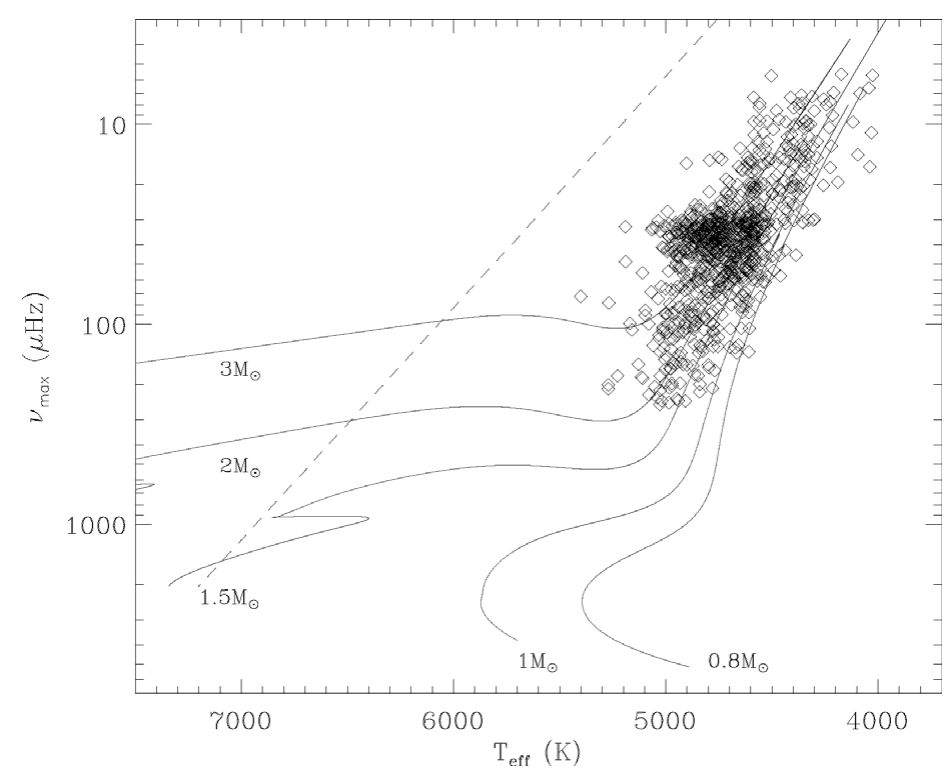

Figure 15. 'Hertzsprung-Russell diagram' of red giants observed with Kepler, using the frequency $\nu_{\max }$ at maximum power as a proxy for luminosity. The curves show evolution tracks for models at the indicated ages. Figure courtesy of D. Huber (see Huber et al. 2010).

To the extent that the modes are trapped in the convective envelope, whose structure is very similar amongst different stars apart from a scaling depending on the stellar mass and radius, one would expect a corresponding similarity between the oscillation frequencies. This is confirmed by the so-called 'universal red-giant oscillation pattern', a term introduced by Mosser et al., of the oscillations (Huber et al. 2010; Mosser et al. 2011). To illustrate this, Fig. 16 shows a normalized and stacked échelle diagram. Here collapsed échelle diagrams for the individual stars, normalized by the large separation, have been stacked after taking out the variation with stellar parameters of the average $\epsilon$ (cf. Eq. 7.1). Clearly there is very little variation with $\nu_{\max }$ and hence stellar properties in the location of the ridges and hence the scaled small separations. This is emphasized by the collapsed version of the diagram in the lower panel; it should be noticed that this also, as indicated, provides a weak indication of modes of degree $l=3$. A lower limit to the width of the ridges is provided by the natural width of the peaks, corresponding to the lifetime of the modes. For $l=0$ and 2 Huber et al. (2010) found a width of around $0.2 \mu \mathrm{Hz}$, essentially independent of the stellar parameters and corresponding to a mode lifetime $\dagger$ of around $18 \mathrm{~d}$. Similar results were obtained by Baudin et al. (2011) based on CoRoT observations. Interestingly, this value of the lifetime is similar to the estimate obtained by Houdek \& Gough (2002) from modelling of $\xi$ Hya.

In Fig. 16 it is evident that the ridge for $l=1$ appears substantially wider than for $l=0$ and 2. This can be understood from the analysis of Dupret et al. (2009), discussed above, which showed that several dipolar modes may reach observable amplitudes in the vicinity of an acoustic resonance (see also Montalbán et al. 2010b). In a major breakthrough in red-giant asteroseismology Beck et al. (2011) and Bedding et al. (2011) demonstrated that the frequencies in such groups of peaks showed clear g-mode-like behaviour; this allowed a determination of the uniform g-mode period spacing (cf. Eq. 5.1).

$\dagger$ defined as the e-folding time of the displacement 


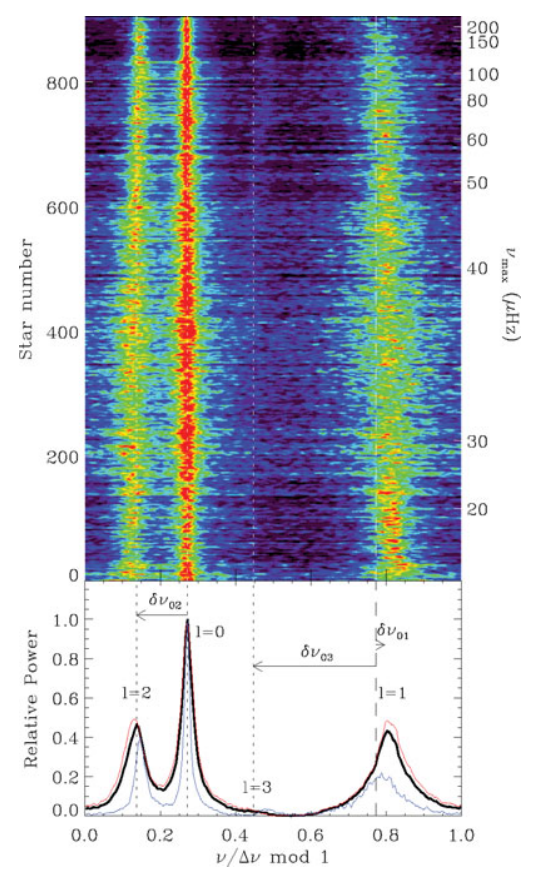

Figure 16. The upper panel shows stacked collapsed, rescaled and shifted échelle diagrams for red giants observed with Kepler. These have been collapsed in the lower panels, where the peaks corresponding to $l=0-3$ are indicated. The thick lines correspond to the full set, and the thin blue and red lines (in the electronic version) correspond to stars with $\nu_{\max }>100 \mu \mathrm{Hz}$ and $\nu_{\max }<50 \mu \mathrm{Hz}$, respectively. Figure courtesy of D. Huber (see Huber et al. 2010).

It was further demonstrated by Bedding et al. (2011) that the inferred value of $\Delta \Pi$ allowed to distinguish between stars on the ascending red-giant branch and stars in the helium-burning clump phase. With further analyses these observations will undoubtedly provide very valuable diagnostics of the central properties of red giants.

The frequencies of purely acoustic modes also contain information beyond the basic parameters $\Delta \nu$ and $\nu_{\max }$. Sharp features in the sound speed lead to systematic departures from the simple asymptotic behaviour in Eq. (7.1), with characteristic properties which provide information about the location and strength of the feature (e.g., Gough 1990). An important example is the effect on the sound speed of the localized reduction in the adiabatic exponent caused by the second ionization of helium, which provides information about the helium abundance (e.g., Vorontsov et al. 1991; Monteiro \& Thompson 2005; Houdek \& Gough 2007). Carrier et al. (2010) and Miglio et al. (2010) found the signature of this effect in the red giant HR 7349 observed by CoRoT. Miglio et al. noted that the inferred location of the second helium ionization zone provided a strong constraint on the properties of the star. Such analyses will undoubtedly be possible for a large number of red giants observed by CoRoT and Kepler.

\section{Concluding remarks}

The last few years have seen amazing progress in the availability of data for asteroseismic investigations of stellar interiors. The full scientific exploitation of these data is just starting. The community is realizing the challenges provided by actual observations, 
compared with the simulations that preceded the missions, and we are hard at work at optimizing the techniques for the data analysis and interpretation, with the goal of addressing specific aspects of stellar interiors. The ability to study solar-like oscillations in hundreds of stars with Kepler has been a positive surprise, allowing comparative studies in ensemble asteroseismology $\dagger$ (Chaplin et al. 2011); however, we still need to extend the investigations to unevolved stars of lower masses, as will surely be possible as ever longer timeseries for individual stars are accumulated. The present authors, at least, had not foreseen the possibility of detailed analyses of the mixed modes in subgiants, providing archaeological information about the properties of the mixed cores during the main-sequence phase (Deheuvels \& Michel 2010). The very recent detection and analysis of features in the pulsations directly related to the g modes in the cores of red giants (Beck et al. 2011; Bedding et al. 2011) have also been a major surprise, with huge potentials both for characterizing the evolutionary state of the stars and for investigating the properties of the deep interiors of these stars. The detection in the Kepler field of a dozen subdwarf B stars showing long-period oscillations, with additional cases being found by CoRoT, is providing tight constraints on the overall properties of stars in this very late phase of evolution and there is certainly a potential for much more detailed investigations. And the list goes on.

We are fortunate currently to have access to three largely complementary space missions with asteroseismic potentials. The MOST mission is perhaps somewhat overshadowed by CoRoT and Kepler, but it continues to provide excellent data on a variety of bright stars with the possibility of selecting targets over a large part of the sky; the recent combined analysis of MOST data and ground-based radial-velocity data for Procyon (Huber et al. 2011) demonstrates the potential of MOST even in the area of solar-like pulsations. CoRoT has fully demonstrated the required sensitivity to study solar-like oscillations in main-sequence stars. The mission has the very substantial advantage of being able to observe both in the direction towards and away from the Galactic centre which allows comparative studies of stellar populations. Also, the stars observed in the asteroseismology field are relatively bright, facilitating the ground-based support observations of these targets, and the CoRoT 'eyes' contain a very broad sample of interesting targets of most types. Finally, Kepler can observe stars for the duration of the mission, optimizing the precision and sensitivity of the observations and allowing the uninterrupted study of potential changes in stellar properties. The heliocentric orbit provides a more stable and quiet environment than the low-earth orbit of CoRoT, in terms of scattered light and magnetospheric disturbances. Also, the Kepler field has been found to be extremely rich in a variety of interesting stars, now to a large extent characterized through the KAI survey phase.

Even so, there is a continued need to improve the observational situation and strong prospects that this will happen. The BRITE Constellation mission, under development by Austria, Canada and Poland (Kuschnig et al. 2009) will fill a very important niche by carrying out photometric observations of the brightest stars across the sky in two colours. On a longer timescale the ESA PLATO mission, if selected, will greatly extend the Kepler results (Catala et al. 2011). As Kepler, PLATO has the dual purpose of exoplanet investigations and asteroseismology. However, PLATO will look at substantially brighter stars in much larger fields. This is important for the crucial ground-based follow-up observations to confirm the detection of a planet in an apparent transit, particularly for earth-size planets which will be a key goal for PLATO as it is for Kepler. Also, as a result PLATO will allow asteroseismic characterization of a substantial fraction of the

$\dagger$ or, according to D. O. Gough, perhaps better synasteroseismology 
stars around which potential planets are found, unlike Kepler where this is an exception. PLATO will be placed in an orbit around the $\mathrm{L}_{2}$ point which shares the advantage, in terms of stability, of Kepler's heliocentric orbit. The planned observing schedule consists of continuous observations of two fields for two or three years each, followed by a 'stopand-stare' phase where each field is observed for a few months. The latter part of the mission will allow investigation of a substantial fraction of the sky, providing a survey of a far more wide-ranging and varied set of stellar pulsations and other types of stellar variability than has been possible even with Kepler.

The great advances provided by the space asteroseismic observations should not blind us to the continued usefulness of ground-based observations. In photometry that allows study of rare objects that may not be available to the space observatories. Also, it provides greater flexibility, e.g., in carrying out observations in several colours, of importance to mode identification. Even more important is the use of ground-based radial-velocity observations, particularly for solar-like oscillations. Observations of solar oscillations from the SOHO mission have clearly demonstrated that the solar background, from granulation and activity, is substantially higher relative to the oscillations for photometric observations than for radial-velocity observations, as already noted by Harvey (1988) (see also Grundahl et al. 2007). This background is evident in the Kepler observations of Gemma illustrated in Fig. 9. This puts a natural limit to the precision and mode selection possible in photometric observations. Thus radial-velocity observations of carefully selected stars are still required to reach the ultimate precision and level of detail in asteroseismic investigations. Such observations can been carried out from the ground, as has been done successfully for a small sample of stars (e.g., Bouchy \& Carrier 2002; Bedding et al. 2004; Bazot et al. 2005; Kjeldsen et al. 2005; Bedding et al. 2007; Arentoft et al. 2008); to reduce the gaps in the data they have been carried out in a coordinated fashion, involving two or more observatories. However, the required state-of-the-art instrumentation is only available for limited periods and certainly not for the month-long observations from several observatories that are needed to secure adequate frequency resolution. This is the motivation for the development of the Stellar Observations Network Group (SONG) network (Grundahl et al. 2009, 2011). SONG is planned to consist of $7-8$ nodes with a suitable geographical distribution in the northern and southern hemisphere. Each node will consist of a $1 \mathrm{~m}$ telescope, equipped with a high-resolution spectrograph for Dopplervelocity observations and a so-called lucky-imaging camera for photometry in crowded fields. With the use of an iodine cell as reference, and with careful optimization of the optics, it is estimated that SONG will be able to study velocity oscillations in a star as the Sun at magnitude 6. The lucky-imaging camera is designed for characterization of exoplanet systems through gravitational micro-lensing (e.g., Dominik et al. 2010). At present a prototype SONG node is under construction with Danish funding, to be placed at the Izaña Observatory on Tenerife, with expected deployment and start of operations in 2011. A Chinese node is being designed and is expected to be operational in 2013, and funding and support for further nodes will be sought through a network of international collaborations.

The data from these projects will provide excellent possibilities for testing present stellar evolution calculations and indicating where improvements should be made. Such improvements are certainly required, particularly when it comes to the treatment of stellar internal dynamics. Impressive progress is being made in the application of complex, yet unavoidably highly simplified, treatments of the interplay between rotation, circulation, and magnetic fields, including also the evolution of stellar internal angular velocity (e.g., Palacios et al. 2003, 2006; Mathis \& Zahn 2004, 2005) (see also Maeder 2009). Indeed, full stellar evolution calculations will undoubtedly require such simplifications in 
the foreseeable future. However, it is equally important that these simplified treatments be tested by detailed simulations of the hydrodynamical phenomena, albeit for conditions that often do not fully reflect the stellar internal conditions. An important example is the simulation of near-surface convection, where computations under reasonably realistic conditions are in fact possible (e.g., Nordlund et al. 2009; Trampedach 2010). Simulations of the deeper parts of the convective envelope and the region below (see Miesch 2005; Miesch \& Toomre 2009, for reviews) unavoidably require simplification, but are providing deep insights into the interaction between convection and rotation, and the generation of magnetic fields (Brun et al. 2004; Browning et al. 2006; Miesch et al. 2008). Such simulations are now being extended to stars other than the Sun (Brun \& Palacios 2009; Brown, B. P., et al. 2011), including the dynamics of stellar convective cores (Brun et al. 2005; Featherstone et al. 2009). The observations by CoRoT, Kepler and projects to follow provide excellent prospects for testing the results of such modelling and hence improve our understanding of stellar internal dynamics.

Acknowledgement: We wish to take this occasion to thank Juri for many years of enjoyable collaboration, as well as for his inspiration and constant friendship. We are very grateful to P. Degroote, J. De Ridder, G. Doğan, D. Huber, T. Kallinger, C. Karoff, K. Kolenberg, R. Szabó and V. Van Grootel for the provision of, or help with, the figures, and to Travis Metcalfe for comments that helped improve the paper. We thank Sacha Brun and Nick Brummell for the excellent organization of an exciting conference, and for their patience with the present authors. The National Center for Atmospheric Research is sponsored by the National Science Foundation (NSF).

\section{References}

Aerts, C., Thoul, A., Daszyńska, J., Scuflaire, R., Waelkens, C., Dupret, M. A., Niemczura, E., \& Noels, A. 2003, Science, 300, 1926

Aerts, C., Christensen-Dalsgaard, J., \& Kurtz, D. W. 2010, Asteroseismology, Springer, Heidelberg

Arentoft, T., Kjeldsen, H., Bedding, T. R., Bazot, M., Christensen-Dalsgaard, J., et al. 2008, ApJ, 687, 1180

Ausseloos, M., Scuflaire, R., Thoul, A., \& Aerts, C. 2004, MNRAS, 355, 352

Auvergne, M., Bodin, P., Boisnard, L., Buey, J.-T., Chaintreuil, S., et al. 2009, A\&A, 506, 411

Baglin, A., Michel, E., \& Auvergne, M. and the CoRoT team 2006, in Proc. SOHO 18 / GONG 2006 / HELAS I Conf. Beyond the spherical Sun, ed. K. Fletcher, ESA SP-624, ESA Publications Division, Noordwijk, The Netherlands

Baglin, A., Auvergne, M., Barge, P., Deleuil, M., \& Michel, E. and the CoRoT Exoplanet Science Team 2009, in Proc. IAU Symp. 253, Transiting Planets, eds F. Pont, D. Sasselov \& M. Holman, IAU and Cambridge University Press, p. 71

Balona, L. A., Pigulski, A., De Cat, P., Handler, G., Gutiérrez-Soto, J., et al. 2011, MNRAS, in the press

Baran, A., Oreiro, R., Pigulski, A., Pérez Hernández, F., Ulla, A., et al. 2009, MNRAS, 392, 1092

Baran, A. S., Kawaler, S. D., Reed, M. D., Quint, A. C., O'Toole, S. J., et al. 2011, MNRAS, in the press. [arXiv:1103.1666v1]

Basu, S., Grundahl, F., Stello, D., Kallinger, T., Hekker, S., et al. 2011, ApJ, 729, L10

Batalha, N. M., Borucki, W. J., Bryson, S. T., Buchhave, L. A., Caldwell, D. A., et al. 2011, ApJ, 729, 27

Baudin, F., Barban, C., Belkacem, K., Hekker, S., Morel, T., et al. 2011, A\&łA, 529, A84

Bazot, M., Vauclair, S., Bouchy, F., \& Santos, N. C. 2005, A\&A, 440, 615

Beck, P. G., Bedding, T. R., Mosser, B., Stello, D., Garcia, R. A., et al. 2011, Science, 332, 205.

Bedding, T. R. 2003, ApESSS, 284, 61 
Bedding, T. R. \& Kjeldsen, H. 2003, PASA, 20, 203

Bedding, T. R., Kjeldsen, H., Butler, R. P., McCarthy, C., Marcy, G. W., O'Toole, S. J., Tinney, C. G., \& Wright, J. T. 2004, ApJ, 614, 380

Bedding, T. R., Kjeldsen, H., Arentoft, T., Bouchy, F., Brandbyge, J., et al. 2007, ApJ, 663, 1315

Bedding, T. R., Huber, D., Stello, D., Elsworth, Y. P., Hekker, S., et al., 2010, ApJ, 713, L176

Bedding, T. R., Mosser, B., Huber, D., Montalbán, J., Beck, P., et al. 2011, Nature, 471, 608

Belkacem, K., Goupil, M. J., Dupret, M. A., Samadi, R., Baudin, F., Noels, A., \& Mosser, B. 2011, $A \mathscr{E} A$, in the press. [arXiv:1104.0630v2]

Blažko, S. 1907, AN, 175, 325

Borucki, W., Koch, D., Bathalha, N., Caldwell, D., Christensen-Dalsgaard, J., et al. 2009, in Proc. IAU Symp. 253, Transiting Planets, eds F. Pont, D. Sasselov \& M. Holman, IAU and Cambridge University Press, p. 289

Borucki, W. J., Koch, D., Basri, G., Batalha, N., Brown, T., et al. 2010, Science, 327, 977

Bouchy, F. \& Carrier, F. 2001, A\&A, 374, L5

Bouchy, F. \& Carrier, F. 2002, A\& $A, 390,205$

Brown, B. P., Miesch, M. S., Browning, M. K., Brun, A. S., \& Toomre, J. 2011, ApJ, 731, 69

Brown, T. M. \& Gilliland, R. L. 1994, ARAA, 32, 37

Brown, T. M., Gilliland, R. L., Noyes, R. W., \& Ramsey, L. W. 1991, ApJ, 368, 599

Brown, T. M., Latham, D. W., Everett, M. E., \& Esquerdo, G. A. 2011, submitted to ApJ. [arXiv: 1102.0342]

Browning, M. K., Miesch, M. S., Brun, A. S., \& Toomre, J. 2006, ApJ, 648, L157

Brun, A. S. \& Palacios, A. 2009, ApJ, 702, 1078

Brun, A. S., Miesch, M. S., \& Toomre, J. 2004, ApJ, 614, 1073

Brun, A. S., Browning, M. K., \& Toomre, J. 2005, ApJ, 629, 461

Carrier, F., De Ridder, J., Baudin, F., Barban, C., Hatzes, A. P., et al. 2010, A\& $\&$, 509, A73

Catala, C. \& Appourchaux, T. and the PLATO Mission Consortium 2011, in Proc. GONGSoHO 24: A new era of seismology of the sun and solar-like stars, ed. T. Appourchaux, J. Phys.: Conf. Ser., 271, 012084

Chadid, M., Perini, C., Bono, G., Auvergne, M., Baglin, A., Weiss, W. W., \& Deboscher, J. 2011, A\&A A 527, A146

Chaplin, W. J., Houdek, G., Elsworth, Y., Gough, D. O., Isaak, G. R., \& New, R. 2005, MNRAS, 360,859

Chaplin, W. J., Appourchaux, T., Elsworth, Y., García, R. A., Houdek, G., et al., 2010, ApJ, 713, L169

Chaplin, W. J., Kjeldsen, H., Christensen-Dalsgaard, J., Basu, S., Miglio, A., et al. 2011, Science, 332,213

Charpinet, S., Fontaine, G., Brassard, P., \& Dorman, B. 1996, ApJ, 471, L103

Charpinet, S., Green, E. M., Baglin, A., van Grootel, V., \& Fontaine, G., et al. 2010, A\&SA, 516, L6

Christensen-Dalsgaard, J. 2002, Rev. Mod. Phys., 74, 1073

Christensen-Dalsgaard, J. 2004, Solar Phys., 220, 137

Christensen-Dalsgaard, J. \& Frandsen, S. 1983, Solar Phys., 82, 469

Christensen-Dalsgaard, J., Kjeldsen, H., \& Mattei, J. A. 2001, ApJ, 562, L141

Christensen-Dalsgaard, J., Arentoft, T., Brown, T. M., Gilliland, R. L., Kjeldsen, H., Borucki, W. J., \& Koch, D. 2008, in Proc. HELAS II International Conference: Helioseismology, Asteroseismology and the MHD Connections, eds L. Gizon \& M. Roth, J. Phys.: Conf. Ser., 118, 012039

Christensen-Dalsgaard, J., Kjeldsen, H., Brown, T. M., Gilliland, R. L., Arentoft, T., Frandsen, S., Quirion, P.-O., Borucki, W. J., Koch, D., \& Jenkins, J. M. 2010, ApJ, 713, L164

Cox, J. P. \& Whitney, C. 1958, ApJ, 127, 561

Degroote, P., Aerts, C., Samadi, R., Miglio, A., Briquet, M., Auvergne, M., Baglin, A., Baudin, F., Catala, C., \& Michel, E. 2010a, AN, 331, 1065

Degroote, P., Aerts, C., Baglin, A., Miglio, A., Briquet, M., et al. 2010b, Nature, 464, 259

Deheuvels, S. \& Michel, E. 2010, AN, 331, 929 
De Ridder, J., Barban, C., Baudin, F., Carrier, F., Hatzes, A. P., et al. 2009, Nature, 459, 398

Dominik, M., Jørgensen, U. G., Rattenbury, N. J., Mathiasen, M., Hinse, T. C., et al. 2010, AN, 331,671

Dupret, M.-A., Belkacem, K., Samadi, R., Montalban, J., Moreira, O., et al. 2009, A\&্A, 506, 57

Dziembowski, W. A. \& Pamyatnykh, A. A. 2008, MNRAS, 385, 2061

Dziembowski, W. A. \& Soszyński, I. 2010, A\& A, 524, A88

Dziembowski, W. A., Gough, D. O., Houdek, G., \& Sienkiewicz, R. 2001, MNRAS, 328, 601

Featherstone, N. A., Browning, M. K., Brun, A. S., \& Toomre, J. 2009, ApJ, 705, 1000

Fletcher, S. T., Chaplin, W. J., Elsworth, Y., Schou, J., \& Buzasi, D. 2006, MNRAS, 371, 935

Fontaine, G., Brassard, P., Charpinet, S., Green, E. M., Chayer, P., Billères, M., \& Randall, S. K. 2003, ApJ, 597, 518

Fontaine, G., Brassard, P., Charpinet, S., \& Chayer, P. 2006, Mem. Soc. Astron. Ital., 77, 49

Frandsen, S., Carrier, F., Aerts, C., Stello, D., Maas, T., et al. 2002, A\&6A, 394, L5

Gai, N., Basu, S., Chaplin, W. J., \& Elsworth, Y. 2011, ApJ, 730, 63

García, R. A., Mathur, S., Salabert, D., Ballot, J., Régulo, C., Metcalfe, T. S., \& Baglin, A. 2010, Science, 329 1032,

Gilliland, R. L., Brown, T. M., Christensen-Dalsgaard, J., Kjeldsen, H., Aerts, C., et al., 2010, PASP, 122, 131

Gizon, L., Birch, A. C., \& Spruit, H. C. 2010, ARAA, 48, 289

Goldreich, P. \& Keeley, D. A. 1977, ApJ, 212, 243

Gough, D. O. 1986, in Hydrodynamic and magnetohydrodynamic problems in the Sun and stars, ed. Y. Osaki, Department of Astronomy, University of Tokyo, p. 117

Gough, D. O. 1990, in Progress of seismology of the sun and stars, Lecture Notes in Physics, vol. 367, eds Y. Osaki \& H. Shibahashi, Springer, Berlin, p. 283

Gough, D. O. \& Toomre, J. 1991, ARAA, 29, 627

Gough, D. O., Kosovichev, A. G., Toomre, J., Anderson, E. R., Antia, H. M., et al. 1996, Science, 272,1296

Green, E. M., Fontaine, G., Reed, M. D., Callerame, K., Seitenzahl, I. R., et al. 2003, ApJ, 583, L31

Grundahl, F., Kjeldsen, H., Christensen-Dalsgaard, J., Arentoft, T., \& Frandsen, S. 2007, Comm. in Asteroseismology, 150, 300

Grundahl, F., Christensen-Dalsgaard, J., Kjeldsen, H., Jørgensen, U. G., Arentoft, T., Frandsen, S., \& Kjærgaard, P. 2009, in Proc. GONG2008/SOHO21 meeting: Solar-stellar Dynamos as revealed by Helio- and Asteroseismology, eds M. Dikpati, T. Arentoft, I. González Hernández, C. Lindsey \& F. Hill, ASP Conf. Ser., 416, San Francisco, p. 579

Grundahl, F., Christensen-Dalsgaard, J., Jørgensen, U. G., Kjeldsen, H., Frandsen, S., \& Kjærgaard Rasmussen, P. 2011, in Proc. GONG-SoHO 24: A new era of seismology of the sun and solar-like stars, ed. T. Appourchaux, J. Phys.: Conf. Ser., 271, 012083

Handler, G., Aerts, C. (and an international team of 50 astronomers) 2004, in Proc. IAU Colloq. No 193: Variable Stars in the Local Group, eds D. W. Kurtz \& K. Pollard, ASP Conf. Ser., 310, San Francisco, p. 221

Harvey, J. W. 1988, in Proc. IAU Symposium No 123, Advances in helio- and asteroseismology, eds J. Christensen-Dalsgaard \& S. Frandsen, Reidel, Dordrecht, p. 497

Heber, U. 2009, ARAA, 47, 211

Hekker, S., Caerts, C., De Ridder, J., \& Carrier, F. 2006, A\&JA, 458, 931

Hekker, S., Kallinger, T., Baudin, F., De Ridder, J., Barban, C., Carrier, F., Hatzes, A. P., Weiss, W. W., \& Baglin, A. 2009, A\& $A, 506,465$

Hekker, S., Gilliland, R. L., Elsworth, Y., Chaplin, W. J., De Ridder, J., Stello, D., Kallinger, T., Ibrahim, K. A., Klaus, T. C., \& Li, J. 2011, MNRAS, in the press. [arXiv:1103.0141]

Houdek, G., Balmforth, N. J., Christensen-Dalsgaard, J., \& Gough, D. O. 1999, A\&A, 351, 582

Houdek, G. \& Gough, D. O. 2002, MNRAS, 336, L65

Houdek, G. \& Gough, D. O. 2007, MNRAS, 375, 861

Huber, D., Bedding, T. R., Arentoft, T., Gruberbauer, M., Guenther, D. B., Houdek, G., Kallinger, T., Kjeldsen, H., Matthews, J. M., Stello, D., \& Weiss, W. W. 2011, ApJ, 94

Huber, D., Bedding, T. R., Stello, D., Mosser, B., Mathur, S., et al. 2010, ApJ, 723, 1607 
Kallinger, T., Weiss, W. W., Barban, C., Baudin, F., Cameron, C., Carrier, F., De Ridder, J., Goupil, M.-J., Gruberbauer, M., Hatzes, A., Hekker, S., Samadi, R., \& Deleuil, M. 2010a, $A \mathscr{E} A, 509, \mathrm{~A} 77$

Kallinger, T., Mosser, B., Hekker, S., Huber, D., Stello, D., et al. 2010b, A\&3A, 522, A1

Karoff, C., Metcalfe, T. S., Chaplin, W. J., Elsworth, Y., Kjeldsen, H., Arentoft, T., \& Buzasi, D. 2009, MNRAS, 399, 914

Kilkenny, D., Koen, C., O'Donoghue, D., \& Stobie, R. S. 1997, MNRAS, 285, 640

Kiss, L. L. \& Bedding, T. R. 2003, MNRAS, 343, L79

Kiss, L. L. \& Bedding, T. R. 2004, MNRAS, 347, L83

Kjeldsen, H. \& Bedding, T. R. 1995, A\&SA, 293, 87

Kjeldsen, H., Bedding, T. R., Viskum, M., \& Frandsen, S. 1995, AJ, 109, 1313

Kjeldsen, H., Bedding, T. R., Butler, R. P., Christensen-Dalsgaard, J., Kiss, L. L., McCarthy, C., Marcy, G. W., Tinney, C. G., \& Wright, J. T. 2005, ApJ, 635, 1281

Kjeldsen, H., Christensen-Dalsgaard, J., Handberg, R., Brown, T. M., Gilliland, R. L., Borucki, W. J., \& Koch, D. 2010, AN, 331, 966

Koch, D. G., Borucki, W. J., Basri, G., Batalha, N. M., Brown, T. M., et al. 2010, ApJ, 713, L79

Kolenberg, K., Smith, H. A., Gazeas, K. D., Elmaslı, A., Breger, M., et al. 2006, A\&্ A, 459, 577

Kolenberg, K. 2008, in Proc. HELAS II International Conference: Helioseismology, Asteroseismology and the MHD Connections, eds L. Gizon \& M. Roth, J. Phys.: Conf. Ser., 118, 012060

Kolenberg, K., Szabó, R., Kurtz, D. W., Gilliland, R. L., Christensen-Dalsgaard, J., et al. 2010, ApJ, 713, L198

Kolenberg, K., Bryson, S., Szabó, R., Kurtz, D. W., Smolec, R., et al. 2011, MNRAS, 411, 878

Kuschnig, R., Weiss, W. W., Moffat, A., \& Kudelka, O. 2009, in Proc. GONG2008/SOHO21 meeting: Solar-stellar Dynamos as revealed by Helio- and Asteroseismology, eds M. Dikpati, T. Arentoft, I. González Hernández, C. Lindsey \& F. Hill, ASP Conf. Ser., 416, San Francisco, p. 587

Lamb, H. 1909, Proc. London Math. Soc., 7, 122

Libbrecht, K. G. \& Woodard, M. F. 1990, Nature, 345, 779

Maeder, A. 2009, Physics, formation and evolution of rotating stars, Springer, Berlin

Mathis, S. \& Zahn, J.-P. 2004, A\&SA, 425, 229 (Erratum: A\&A, 462, 1063; 2007)

Mathis, S. \& Zahn, J.-P. 2005, A\&A, 440, 653

Matthews, J. M. 2007, Comm. in Asteroseismology, 150, 333

Metcalfe, T. S., Dziembowski, W. A., Judge, P. G., \& Snow, M. 2007, MNRAS, 379, L16

Metcalfe, T. S., Monteiro, M. J. P. F. G., Thompson, M. J., Molenda-Żakowicz, J., Appourchaux, T., et al. 2010, ApJ, 723, 1583

Michel, E., Baglin, A., Weiss, W. W., Auvergne, M., Catala, C., et al. 2008a, Comm. in Asteroseismology, 156, 73

Michel, E., Baglin, A., Auvergne, M., Catala, C., Samadi, R., et al. 2008b, Science, 322, 558

Miesch, M. S. 2005, Living Rev. Solar Phys., 2, 1. URL (cited on 29/3/11): http://www.livingreviews.org/lrsp-2005-1

Miesch, M. S. \& Toomre, J. 2009, ARFM, 41, 317

Miesch, M. S., Brun, A. S., DeRosa, M. L., \& Toomre, J. 2008, ApJ, 673, 557

Miglio, A., Montalbàn, J., Noels, A., \& Eggenberger, P. 2008, MNRAS, 386, 1487

Miglio, A., Montalbán, J., Baudin, F., Eggenberger, P., Noels, A., Hekker, S., De Ridder, J., Weiss, W., \& Baglin, A. 2009, A\& $A, 503$, L21

Miglio, A., Montalbán, J., Carrier, F., De Ridder, J., Mosser, B., Eggenberger, P., Scuflaire, R., Ventura, P., D’Antona, F., Noels, A., \& Baglin, A. 2010, A\&4A, 520, L6

Montalbán, J., Miglio, A., Noels, A., Scuflaire, R., \& Ventura, P. 2010a, ApJ, 721, L182

Montalbán, J., Miglio, A., Noels, A., Scuflaire, R., \& Ventura, P. 2010b, AN, 331, 1010

Monteiro, M. J. P. F. G. \& Thompson, M. J. 2005, MNRAS, 361, 1187

Moskalik, P. \& Dziembowski, W. A. 1992, A\& A, 256, L5

Mosser, B., Belkacem, K., Goupil, M.-J., Miglio, A., Morel, T., et al. 2010, A\& A, 517, A22

Mosser, B., Belkacem, K., Goupil, M. J., Michel, E., \& Elsworth, Y., et al. 2011, A 3 A, 525, L9 
Nordlund, Å., Stein, R. F., \& Asplund, M. 2009, Living Rev. Solar Phys., 6, 2. URL (cited on 29/3/11): http://www. livingreviews . org/lrsp-2009-2

Østensen, R. H., Silvotti, R., Charpinet, S., Oreiro, R., \& Handler, G., et al. 2010, MNRAS, 409,1470

Østensen, R. H., Silvotti, R., Charpinet, S., Oreiro, R., \& Bloemen, S., et al. 2011, MNRAS, in the press. [arXiv:1101.4150]

Palacios, A., Talon, S., Charbonnel, C., \& Forestini, M. 2003, A\& A, 399, 603

Palacios, A., Charbonnel, C., Talon, S., \& Siess, L. 2006, A\&A, 453, 261

Pamyatnykh, A. A. 1999, AcA, 49, 119

Pamyatnykh, A. A., Handler, G., \& Dziembowski, W. A. 2004, MNRAS, 350, 1022

Poretti, E., Paparó, M., Deleuil, M., Chadid, M., \& Kolenberg, K., et al. 2010, A\& A, 520, A108

Randall, S. K., Green, E. M., Fontaine, G., Brassard, P., \& Kilkenny, D., et al. 2006a, ApJ, 643, 1198

Randall, S. K., Green, E. M., Fontaine, G., Brassard, P., Terndrup, D. M., Brown, N., Fontaine, M., Zacharias, P., \& Chayer, P. 2006b, ApJ, 645, 1464

Reed, M. D., Kawaler, S. D., Østensen, R. H., Bloemen, S., Baran, A., et al. 2010, MNRAS, 409, 1496

Reed, M. D., Baran, A., Quint, A. C., Kawaler, S. D., O'Toole, S. J., et al. 2011, MNRAS, in the press. [arXiv:1102.4286 [astro-ph.SR]]

Salaris, M., Cassisi, S., \& Weiss, A. 2002, PASP, 114, 375

Smith, H. A. 1995, RR Lyrae stars, Cambridge Astrophysics Series, Cambridge University Press

Soszyński, I., Dziembowski, W. A., Udalski, A., Kubiak, M., Szymański, M. K., Pietrzyński, G., Wyrzykowski, Ł., Szewczyk, O., \& Ulaczyk, K. 2007, AcA, 57, 201

Stello, D., Bruntt, H., Preston, H., \& Buzasi, D. 2008, ApJ, 674, L53

Stello, D., Basu, S., Bruntt, H., Mosser, B., Stevens, I. R., et al. 2010, ApJ, 713, L182

Szabó, R., Kolláth, Z., Molnár, L., Kolenberg, K., Kurtz, D. W., et al. 2010, MNRAS, 409, 1244

Tassoul, M. 1980, ApJ Suppl., 43, 469

Trampedach, R. 2010, ApESSS, 328, 213

Van Grootel, V., Charpinet, S., Fontaine, G., Brassard, P., Green, E. M., et al. 2010a, ApJ, 718, L97

Van Grootel, V., Charpinet, S., Fontaine, G., Green, E. M., \& Brassard, P. 2010b, A\&SA, 524, A63

Verner, G. A., Elsworth, Y., Chaplin, W. J., Campante, T. L., Corsaro, E., et al. 2011, submitted to MNRAS

Vorontsov, S. V., Baturin, V. A., \& Pamyatnykh, A. A. 1991, Nature, 349, 49

Walker, G., Matthews, J., Kuschnig, R., Johnson, R., Rucinski, S., et al. 2003, PASP, 115, 1023

Woodard, M. F. \& Noyes, R. W. 1985, Nature, 318, 449 